\title{
Decay to Equilibrium in Random Spin Systems on a Lattice
}

\author{
Alice Guionnet ${ }^{1}$, Boguslaw Zegarlinski ${ }^{2}$ \\ ${ }^{1}$ Mathematiques, CNRS URA 0743, Universite Paris-Sud Orsay 91405, France. \\ E-mail: guionnet@stats.matups.fr \\ ${ }^{2}$ Department of Mathematics, Imperial College London SW7 2BZ, UK. E-mail: boz@ic.ac.uk
}

Received: 1 January 1996/Accepted: 5 May 1996

\begin{abstract}
We study continuous and discrete spin systems on a lattice with random interactions of finite range. In particular for sufficiently large interactions we prove no spectral gap property in the high temperature region. Moreover we show that in two dimensions, if the temperature is sufficiently high and the probability of interaction to be large is small enough, we have an almost sure stretched exponential upper bound for the decay to equilibrium.
\end{abstract}

\section{Introduction}

It is known that the spin systems with random interactions can exhibit a rather unusual behaviour even in the high temperature equilibrium, [21, 13]. This equilibrium behaviour has been extensively studied on the rigorous level, (see e.g. $[5,18,7$, $6,25,20,14,15, \ldots, 40]$ and references given there), and at the moment we have relatively good understanding of the high temperature phase. In particular it is known that for random spin systems with short range interactions with probability one we have an exponential decay of correlations with nonrandom characteristic length. The understanding of the corresponding stochastic dynamics on the rigorous level is much less advanced, although recently some progress has been made. In the direction of mean field models, the asymptotics of Langevin dynamics have been investigated in $[2,3,4,23]$ and have been proven to exhibit unusual non-Markovian features. For short range models, it has been shown in [39] that the generator of Glauber dynamics for the Ising type discrete spin systems on $\mathbb{Z}^{d}$ cannot have a spectral gap with probability one, if the couplings are allowed to take sufficiently large values. This is in distinction to nonrandom spin systems where the spectral gap is a typical feature of the high temperature phase, (see e.g. [1,33-35] and references given there). The no spectral gap result of [39] has been strongly based on the finite volume estimates of the spectral gap in the phase transition region obtained in [37]. In the present paper we extend the result of [39] to systems where weaker information is available, including an interesting case of continuous spins. Such a 
property suggests that we can no longer expect to have an exponential decay to equilibrium for local variables. Indeed it has been proven in [39] that for the one dimensional spin systems with unbounded interactions, for which the couplings satisfy some exponential bound, one has with probability one an upper bound for the decay to equilibrium in uniform norm for local observables given by a stretched exponential. More recently, a very interesting result about the high temperature decay to equilibrium for random spin systems on $\mathbb{Z}^{d}, d \geqq 1$ has been obtained in [20]. Using some clever percolation technique, the authors have shown an almost sure upper bound for the decay to equilibrium which is faster than any polynomial, but slower than a stretched exponential.

In the present paper we study further the problem of decay to equilibrium in disordered spin systems with short range interactions, but including also continuous spin systems. For such systems there is no similar percolation technique available. We use a strategy, (originated in [24], see also [33-36 and 39]), based on application of local hypercontractivity. For such a strategy to work one needs to have a stochastic dynamics with a good approximation property by finite volume stochastic dynamics. In Sect. 2 we show how to extend the classical construction (used e.g. in [34 and 27]) to the case when the diffusion coefficients, respectively in the discrete case when the rate functions, are possibly unbounded with probability one. In general the finite volume approximation can be rather slow. We show however that there is a large class of interactions with unbounded couplings for which one has an exponential approximation property up to linear times (in the size of the volume). Distinguishing this class is important when we want to study non-exponential decay to equilibrium, (although in view of no spectral gap property shown in Sect. 3, the class of interactions with uniformly bounded but sufficiently large couplings is already interesting). In Sects. 4 and 5 we show that in such class (including unbounded couplings) when the dimension of the lattice is two, one has a stretched exponential upper bound on the decay to equilibrium with probability one in the high temperature region. This result is true for continuous as well as for discrete spins. The main technical ingredient is the almost sure control on the growth of the logarithmic Sobolev coefficient for finite volume Gibbs measures. It is presented in Sect. 5, in the discrete setting; the continuous one is easier. We show that this coefficient is growing in a sublinear way and thus one gets a stretched exponential upper bound for the decay to equilibrium. As the temperature is increasing one has less and less stretching and, in the case of unbounded couplings, in the limit when the temperature goes to infinity, we approach the exponential decay; for bounded couplings one gets the exponential decay above a finite temperature, which clearly suggests some dynamical phase transition. We can show that also in some higher dimensional disordered systems the almost sure upper bound for the decay is faster than the one obtained in [20]. To keep the size of this paper reasonable, we decided to present that elsewhere.

The no spectral gap property and the almost sure upper bounds for the decay to equilibrium suggest that in disordered spin systems there is a qualitative difference between the decay to equilibrium and the decay of correlations (which in systems with the finite speed of propagation of information gives us always the lower bound). It is also known that for the disordered systems in the high temperature region we have no analyticity properties. This would somehow confirm a hypothesis (advocated in [34], see also $[28,29,32])$ that the exponential decay to equilibrium in the uniform norm is related to analytic behaviour of the system. 
1.1. Preliminaries. Let $\mathbb{Z}^{d}$ be a $d \in \mathbb{N}$ dimensional integer lattice with a metric $d(i, j) \equiv \sum_{\alpha=1}^{d}\left|i^{\alpha}-j^{\alpha}\right|, j, i \in \mathbb{Z}^{d}$. By $\mathscr{F}$ we denote the family of all finite subsets of the lattice. Let $\mathscr{F}_{0} \equiv\left\{\Lambda_{n} \in \mathscr{F}\right\}_{n \in \mathbb{N}}$ be a countable exhaustion of the lattice, i.e. an increasing sequence of finite sets invading all the lattice. We will assume that $\mathscr{F}_{0}$ is a Fisher sequence, [31]. A cardinality (the volume) of a finite set $X \in \mathscr{F}$ will be denoted by $|X|$. Given a positive number $R$ we define an $R$-boundary of a set $\Lambda \subset \mathbb{Z}^{d}$ as follows:

$$
\partial_{R} \Lambda \equiv\left\{j \in \Lambda^{c}: d(j, \Lambda) \leqq R\right\}
$$

where $\Lambda^{c} \equiv \mathbb{Z}^{d} \backslash \Lambda$ and $d(j, \Lambda)$ denotes the distance of $j$ from the set $\Lambda$.

Dependent on the context, our single spin space $\mathbf{M}$ will be either a finite set or a smooth, compact, connected (finite dimensional) Riemannian manifold. In the first and the second situation we will speak about the discrete and continuous cases, respectively. The typical examples frequently used in statistical mechanics are given by a two point set $\{-1,+1\}$ and an $N$-dimensional unit sphere $S^{N} \subset \mathbb{R}^{N+1}$, respectively. A configuration space of the infinite spin system on the lattice $\mathbb{Z}^{d}$ is a product space $\Omega \equiv \mathbf{M}^{\mathbb{Z}^{d}}$ with a Borel $\sigma$-algebra $\Sigma$ given by the product topology. Given a finite set $X \in \mathscr{F}$ we define a projection

$$
\Omega \ni \omega \mapsto \omega_{X} \in \mathbf{M}^{X},
$$

in particular if $X \equiv\{j\}$ is a single point set, such a projection map is called a spin at site $j \in \mathbb{Z}^{d}$ and is denoted by $\sigma_{j}$. Given a set $X \in \mathscr{F}$, we define a measurable mapping

$$
\Omega \times \Omega \ni(\omega, \tilde{\omega}) \mapsto \omega \bullet_{X} \tilde{\omega} \in \Omega
$$

by setting

$$
\left(\omega \bullet \bullet_{X} \tilde{\omega}\right)_{j}=\left\{\begin{array}{ll}
\omega_{j} & \text { if } j \in X \\
\tilde{\omega}_{j} & \text { otherwise }
\end{array} .\right.
$$

In the case when $X=\{j\}$ is a one point set, we will simplify this notation by writing $\omega \bullet_{j} \tilde{\omega} \equiv \omega \bullet_{\{j\}} \tilde{\omega}$. We introduce also a measurable mapping

$$
\mathbf{M}^{X} \times \mathbf{M}^{X^{c}} \ni\left(y_{X}, z_{X^{c}}\right) \mapsto y_{X} \circ_{X} z_{X^{c}} \in \Omega
$$

by setting

$$
\left(y_{X} \circ_{X} z_{X^{c}}\right)_{j}=\left\{\begin{array}{ll}
y_{j} & \text { if } j \in X \\
z_{j} & \text { otherwise }
\end{array} .\right.
$$

For $\Lambda \subset \mathbb{Z}^{d}$ we define $\Sigma_{\Lambda}$ as a smallest $\sigma$-algebra for which all the spins $\sigma_{j}, j \in \Lambda$ are measurable. A set of continuous and bounded measurable functions on $(\Omega, \Sigma)$ will be denoted by $\mathscr{C} \equiv \mathscr{C}(\Omega)$ and $\mathbb{B} \equiv \mathbb{B}(\Omega)$, respectively. By $\|\cdot\|_{u}$ we denote the supremum norm on $\mathbb{B}$. A measurable or continuous real function $f$ on the configuration space is called a local function if there is a finite set $Y \in \mathscr{F}$ such that $f$ is $\Sigma_{Y}$ measurable. This means that there is a measurable (or continuous) function $\varphi_{Y}$ on $\mathbf{M}^{Y}$ such that $f(\omega)=\varphi_{Y}\left(\omega_{Y}\right)$. A set of all local continuous and local measurable functions will be denoted by $\mathscr{C}_{0} \equiv \mathscr{C}_{0}(\Omega)$ and $\mathbb{B}_{0} \equiv \mathbb{B}_{0}(\Omega)$, respectively. For a given local function $f$, a smallest set $Z \in \mathscr{F}$ for which $f$ is $\Sigma_{Z}$ measurable will be denoted by $\Lambda(f)$ and called a set of localisation of the function $f$. Frequently it will be convenient to use the following notation $d(f, Y) \equiv d(\Lambda(f), Y)$, where on 
the right-hand side we have the distance between two subsets $\Lambda(f)$ and $Y$ of the lattice $\mathbb{Z}^{d}$.

By $v_{\mathbf{M}}$ we will denote a probability measure on $\left(\mathbf{M}, \mathscr{B}_{\mathbf{M}}\right)$ given by a normalised counting measure or the normalised Riemannian volume corresponding to a given Riemannian metric, respectively. A product measure $\mu_{0} \equiv v_{\mathbf{M}}^{\otimes \mathbb{Z}^{d}}$ on $(\Omega, \Sigma)$ is called a free measure. We will use a notation $\mu f$ to denote an expectation value of measurable function $f$ and a probability measure $\mu$ given on the same measurable space (whenever it is well defined). A covariance of two functions $f$ and $g$ with the measure $\mu$ will be denoted by

$$
\mu(f, g) \equiv \mu f g-\mu f \mu g .
$$

In the configuration space $\Omega$ we have a natural representation $\left(T_{j}\right)_{j \in \mathbb{Z}^{d}}$ of the group of translations of the lattice given by the following measurable maps:

$$
\left(T_{j} \omega\right)_{k} \equiv \omega_{k-j}
$$

We can use it to induce a representation on the space of measurable functions by setting

$$
\left(\mathbf{T}_{j} f\right)(\omega) \equiv f\left(T_{j} \omega\right) .
$$

Given a measurable function $f$ on $(\Omega, \Sigma)$, for every configuration $\omega \in \Omega$ and a finite set $X \in \mathscr{F}$, we define a function

$$
\mathbf{M}^{X} \ni y \mapsto f_{X}(y \mid \omega) \equiv f\left(y \circ_{X} \omega_{X^{c}}\right) .
$$

In the particular case of the one point set $X=\{j\}$ we use a simplified notation $f_{j}(y \mid \omega) \equiv f\left(y \circ_{j} \omega\right) \equiv f_{\{j\}}(y \mid \omega)$. In the continuous case, if for every $j \in \mathbb{Z}^{d}$ and $\omega$, the function $f_{j}(\cdot \mid \omega)$ is differentiable, we say that $f$ is differentiable. For such a function we introduce its gradient $\nabla_{\Lambda} f \equiv\left(\nabla_{j} f\right)_{j \in \Lambda}$, with respect to coordinates in a set $\Lambda \subseteq \mathbb{Z}^{d}$, where

$$
\nabla_{j} f(\omega) \equiv \partial_{\mathbf{M}} f_{j}(x \mid \omega)_{\mid x=\omega_{j}}
$$

with $\partial_{\mathbf{M}}$ denoting the gradient operator on the Riemannian manifold $\mathbf{M}$. Using the scalar product $(\cdot \mid \cdot)_{j}$ associated to the Riemannian manifold $\mathbf{M}$ (at site $i$ ), we introduce the square of the gradient $\nabla_{\Lambda} f$ by the following formula:

$$
\left|\nabla_{\Lambda} f\right|^{2}(\omega) \equiv \sum_{j \in \Lambda}\left(\nabla_{j} f(\omega) \mid \nabla_{j} f(\omega)\right)_{j}
$$

If $\Lambda=\mathbb{Z}^{d}$, for simplicity we will omit the corresponding index in the notation of the gradient. The space of all differentiable functions for which the following seminorm is finite

$$
\||f|\| \equiv \sum_{j \in \mathbb{Z}^{d}}\left\|\left|\nabla_{j} f\right|\right\|_{u}
$$

will be denoted by $\mathscr{C}^{1}$. Inductively, we say that a function $f$ is $n$-times differentiable if for every finite set $X \in \mathscr{F}$, and $\omega \in \Omega$ the corresponding function $f_{X}(\cdot \mid \omega)$ on $\mathbf{M}^{X}$ is $n$-times differentiable. Frequently we will use also the following abbreviated notation:

$$
\left\|\nabla_{j} f\right\| \equiv\left\|\left|\nabla_{j} f\right|\right\|_{u}
$$


and

$$
\left\|\nabla_{i} \nabla_{j} f\right\| \equiv\left\|\left|\nabla_{i} \nabla_{j} f\right|\right\|_{u} .
$$

In the discrete case we will use a similar notation, with $\nabla_{j}$ denoting the discrete gradient defined by the formula

$$
\nabla_{j} f(\omega) \equiv f(\omega)-\int_{\mathbf{M}} v_{\mathbf{M}}(d x) f\left(x \circ_{j} \omega_{\{j\}^{c}}\right) .
$$

Let $\left(\mathbb{J}, \mathscr{B}_{\mathbb{J}}, \mathbb{E}\right)$ be a probability space. A potential is a family $\Phi \equiv\left(\Phi_{X}\right)_{X \in \mathscr{F}}$ consisting of measurable functions $\Phi_{X}: \mathbb{J} \times \Omega \rightarrow \mathbb{R}$, such that:

- for every $X \in \mathscr{F}$, the function $\Phi_{X}(\mathbf{J}, \cdot)$ is continuous and $\Sigma_{X}$ measurable (i.e. depends only on the coordinates $\left.\left(\omega_{j}, j \in X\right)\right)$, and

- for every $i \in \mathbb{Z}^{d}$ we have

$$
\sum_{\substack{X \in \mathscr{F} \\ X \ni i}}\left\|\Phi_{X}(\mathbf{J}, \cdot)\right\|_{u}<\infty
$$

- The family $\left\{\Phi_{X}(\cdot, \omega), X \in \mathscr{F}\right\}$ of random variables is mutually independent on the probability space $\left(J, \mathscr{B}_{\mathbb{J}}, \mathbb{E}\right)$. Moreover, the random variables $\Phi_{X+j}\left(\cdot, T_{j} \omega\right)$ and $\Phi_{X}(\cdot, \omega)$ are identically distributed.

Example $A$. Let $\mathbf{J}=\left\{J_{X} \in \mathbb{R}\right\}_{X \in \mathscr{F}}$ be a collection of mutually independent (possibly unbounded) random variables, such that $J_{X}$ and $J_{X+j}$ are identically distributed for any $X \in \mathscr{F}$ and $j \in \mathbb{Z}^{d}$. We set

$$
\Phi_{X}=J_{X} \varphi_{X}, \quad X \in \mathscr{F},
$$

where $\varphi \equiv\left\{\varphi_{X}\right\}_{X \in \mathscr{F}}$ is a nonrandom Gibbsian potential on $(\Omega, \Sigma)$ and $J_{X}$ satisfies some moment conditions, [38]. In discrete case we would get a random spin-type interaction by setting $\varphi_{X}=\sigma_{X} \equiv \prod_{i \in X} \sigma_{i}, X \in \mathscr{F}$.

Example B. Let $\mathbf{M}=S^{N}$ and let $\mathbf{J} \equiv\left\{J_{X} \in \mathbb{R}\right\}_{X \in \mathscr{F}}$ be similar as in the previous example. Let $\rho \equiv\left\{\rho_{X} \in \mathbb{R}\right\}_{X \in \mathscr{F}}$ be a sequence such that

$$
\|\rho\|_{1} \equiv \sup _{i \in \mathbb{Z}^{d}} \sum_{\substack{X \in \mathscr{F} \\ X \ni i}}\left|\rho_{X}\right|<\infty
$$

but

$$
\|\rho\|_{2} \equiv \sup _{i \in \mathbb{Z}^{d}} \sum_{\substack{X \in \mathscr{F} \\ X \ni i}}|X| \cdot\left|\rho_{X}\right|=\infty .
$$

For a fixed vector $v \in S^{N}$ we define

$$
\Phi_{X}(\mathbf{J}, \omega)=\rho_{X} \cos \left(J_{X} \sum_{i \in X} \sigma_{i} \cdot v\right) .
$$

Here $\sigma_{i}$ and $v$ denote unit vectors in $\mathbb{R}^{N+1}$ and the dot the Euclidean scalar product in $\mathbb{R}^{N+1}$. In the present situation our potential $\Phi$ is bounded, but the considerations of [17], (see also [8]), for nonrandom potentials suggest that dependent on the behaviour of moments of $J_{X}, X \in \mathscr{F}$, we can have qualitatively different behaviour at high temperatures. In general one would expect to have unique high temperature 
phase, (but for random variables with variance $\operatorname{var}\left(J_{X}\right)$ decreasing slower to zero than $1 / \sqrt{|X|}$ it is a hard open problem).

In this paper we restrict ourselves to potentials of finite range, i.e. we assume that there is a positive integer $R$ such that $\Phi_{X} \equiv 0$ whenever $\operatorname{diam}(X) \geqq R$. Additionally in the continuous case we assume that the potential is twice differentiable, in the sense that for every $X \in \mathscr{F}$ the corresponding function $\Phi_{X}(\mathbf{J}, \cdot)$ is twice differentiable, for every $\mathbf{J} \in \mathbb{J}$.

Given a potential $\Phi$, we define interaction energy $U_{\Lambda}$ and Hamiltonian $H_{\Lambda}$ in a finite set $\Lambda$, respectively by

$$
U_{\Lambda}(\mathbf{J}, \omega) \equiv \sum_{\substack{X \in \mathscr{F} \\ X \cap \Lambda \neq \emptyset}} \Phi_{X}(\mathbf{J}, \omega)
$$

and

$$
H_{\Lambda}(\mathbf{J}, \omega) \equiv \sum_{X \subseteq \Lambda} \Phi_{X}(\mathbf{J}, \omega)
$$

We introduce finite volume Gibbs measures $\mu_{\Lambda}^{\mathbf{J}, \omega}$ and $\mu_{\mathbf{J}, \Lambda}^{0}$ in a finite volume $\Lambda$ with external conditions given by configuration $\omega \in \Omega$ and with zero boundary conditions, respectively, by

$$
\mu_{\mathbf{J}, \Lambda}^{\omega}(f) \equiv \frac{1}{Z_{\Lambda}(\mathbf{J}, \omega)} \int_{\mathbf{M}^{\Lambda}} \mu_{0}^{\Lambda}\left(d x_{\Lambda}\right) e^{-\beta U_{\Lambda}\left(\mathbf{J}, x_{\Lambda} \circ \omega_{\Lambda^{c}}\right)} f\left(x_{\Lambda} \circ \omega_{\Lambda^{c}}\right)
$$

and

$$
\mu_{\mathbf{J}, \Lambda}^{0}(f) \equiv \frac{1}{Z_{\Lambda}(\mathbf{J}, 0)} \int_{\mathbf{M}^{\Lambda}} \mu_{0}^{\Lambda}\left(d x_{\Lambda}\right) e^{-\beta H_{\Lambda}\left(\mathbf{J}, x_{\Lambda}\right)} f\left(x_{\Lambda}\right)
$$

for local functions $f$, in the second case satisfying $\Lambda(f) \subseteq \Lambda$, where $\mu_{0}^{\Lambda}$ denotes the restriction of the free measure $\mu_{0}$ to $\Sigma_{\Lambda}$.

\section{The Approximation Property for Stochastic Dynamics}

In the continuous case we define a Markov generator $\mathbf{L}_{\Lambda} \equiv \mathbf{L}_{\mathbf{J}, \Lambda}$ in a finite volume $\Lambda \in \mathscr{F}$ by setting

$$
\mathbf{L}_{\Lambda} \equiv \sum_{i \in \mathbb{Z}^{d}} \mathbf{L}_{i}^{\Lambda}
$$

where

$$
\mathbf{L}_{i}^{\Lambda} \equiv \mathbf{L}_{\mathbf{J}, i}^{\Lambda} \equiv \Delta_{i}-\nabla_{i} H_{i, \Lambda} \cdot \nabla_{i}
$$

with $\Delta_{i}$ denoting the Laplace-Beltrami operator and

$$
H_{i, \Lambda} \equiv \sum_{\substack{X \subseteq \Lambda \\ X \ni i}} \Phi_{X}
$$

Note that when the interaction is of finite range $R$, for any $i \in \Lambda$ satisfying $d\left(i, \Lambda^{c}\right) \geqq R$ we have

$$
\mathbf{L}_{i}^{\Lambda}=\mathbf{L}_{i} \equiv \Delta_{i}-\nabla_{i} U_{i} \cdot \nabla_{i}
$$

(Whenever the single spin space $\mathbf{M}$ have a nonempty boundary $\partial \mathbf{M}$, one needs to specify the boundary conditions for the functions in the domain of our Markov 
generator.) Then for any finite set $\Lambda$ and any $\mathbf{J} \in \mathbb{J}$, we have a well defined Markov semigroup $P_{t}^{\Lambda} \equiv P_{t}^{\mathbf{J}, \Lambda} \equiv e^{t \mathbf{L}_{\Lambda}}$. We will be interested in the construction and study of the infinite volume semigroup $P_{t} \equiv P_{t}^{\mathbf{J}}$ defined formally by an operator

$$
\mathbf{L} \equiv \mathbf{L}_{\mathbf{J}} \equiv \sum_{i \in \mathbb{Z}^{d}} \mathbf{L}_{i}
$$

which is well defined on the dense in $\mathscr{C}^{1}$ subset of smooth cylinder functions (satisfying some boundary conditions if $\partial \mathbf{M}$ is nonempty). Moreover such an operator is symmetric and nonnegative in $\mathbb{L}_{2}\left(\mu_{\mathbf{J}}\right)$ for any Gibbs measure $\mu_{\mathbf{J}}$ corresponding to the same interactions. (Following [41], one could also consider nonsymmetric generators.)

In the discrete case we introduce the finite volume generators by a formula

$$
\mathscr{L}_{\Lambda} \equiv \mathscr{L}_{\mathbf{J}, \Lambda} \equiv \sum_{i \in \Lambda} \mathbf{a}_{X+l} \mathscr{L}_{X+i}
$$

where

$$
\mathscr{L}_{X+i} f(\omega) \equiv \mathscr{L}_{\mathbf{J}, X+i} f(\omega) \equiv \mu_{X+i}^{\omega} f-f(\omega)
$$

and $\mathbf{a}_{X+i} \equiv \mathbf{a}_{X+i}(\mathbf{J}, \omega)$ is a positive bounded (but not necessarily uniformly in $\mathbf{J}$ and $X+i), \Sigma_{X^{c}+i}$-measurable function. Frequently the corresponding case when all $\mathbf{a}_{X+j}$ are equal to one and $Z_{X+j}^{\omega}$ is called a heat-bath and Monte-Carlo dynamics, respectively. The corresponding finite volume semigroup $\mathscr{P}_{t}^{X, \Lambda} \equiv \mathscr{P}_{t}^{\mathbf{J}, X, \Lambda}$ is well defined for every $\mathbf{J} \in \mathbf{J}$. We will be interested in the construction and study of an infinite volume semigroups $\mathscr{P}_{t}^{J, X}$ defined formally by a generator

$$
\mathscr{L} \equiv \mathscr{L}_{\mathbf{J}} \equiv \sum_{j \in \mathbb{Z}^{d}} \mathscr{L}_{X+j}
$$

which is well defined on $\mathbb{B}_{0}$ and as an operator in $\mathbb{L}_{2}\left(\mu_{\mathbf{J}}\right)$, with $\mu_{\mathbf{J}}$ being a Gibbs measure corresponding to the same interaction, is nonnegative and symmetric (i.e. satisfies the detailed balance condition).

We will need to show that under some suitable conditions the sequence of finite volume semigroups converges to an infinite volume Markov semigroup, for $\mathbb{E}$-a.a. configurations $\mathbf{J} \in \mathbf{J}$. For further study of the decay to equilibrium we will need also to have a control on such a finite volume approximation.

Theorem 2.1. Suppose $\Phi \equiv\left\{\Phi_{X}\right\}_{X \in \mathscr{F}}$ is a (smooth) interaction of finite range $R$ and there is an $K>d$ such that

Then the limit

$$
\sup _{X \in \mathscr{F}, i, j \in \mathbb{Z}^{d}} \mathbb{E}\left\|\nabla_{i} \nabla_{j} \Phi_{X}\right\|^{K}<\infty .
$$

exists $\mathbb{E}$-a.e..

$$
P_{t}^{\mathbf{J}} f \equiv \lim _{\mathscr{F}_{0}} P_{t}^{\mathbf{J}, \Lambda} f
$$

Moreover for any cube $\Lambda$, any $A \in \mathbb{R}^{+}$and any local function $f \in \mathscr{C}^{1}$ we have

$$
\left\|P_{t}^{\mathbf{J}} f-P_{t}^{\mathbf{J}, \Lambda} f\right\|_{u} \leqq e^{-A t} D(\mathbf{J})\|\| f\|\|
$$

provided that $d\left(f, \Lambda^{c}\right) \geqq \bar{L}$ for some sufficiently large constant $\bar{L} \equiv \bar{L}(\Lambda(f), R, \mathbf{J})$ and

$$
d\left(f, \Lambda^{c}\right)^{1-\delta} \geqq C t
$$

with a constant $C \in \mathbb{R}^{+}$dependent on $\mathbf{J}, \Lambda(f)$ and the choice of $A$, and with some $\delta \in(0,1)$ such that $K \delta>d$. 
Remark. As the formal infinite volume generator $\mathbf{L}_{\mathbf{J}}$ given in (1) is a densely defined nonnegative and symmetric in $\mathbb{L}_{2}\left(\mu_{\mathbf{J}}\right)$, for any Gibbs measure $\mu_{\mathbf{J}}$ corresponding to the same interaction, its Friedrichs extension defines an infinite volume Markov semigroup, without any extra restrictions on the distribution $\mathbb{E}$. Such a semigroup may not be Fellerian and thus a decay to equilibrium in general could not be discussed on the space of continuous functions. One can make similar remarks about the discrete case.

Proof. The proof follows a rather standard line, as e.g. in [34, 27]. Additionally now we need to take care of the $\mathbb{E}$ almost sure behaviour of the nontranslation invariant interaction.

Let $\mathscr{F}_{0} \equiv\left\{\Lambda_{n}\right\}_{n \in \mathbb{N}}$ be a countable exhaustion of the lattice consisting of a cube of size $n$ and centred at the origin. We choose in $\mathbb{Z}^{d}$ a lexicographic order compatible with $\mathscr{F}_{0}$, which interpolates between consecutive cubes by filling the faces. We want to show that for any local function $f \in \mathscr{C}^{1}$ the sequence $P_{t}^{\mathbf{J}, \Lambda_{n}} f, \Lambda_{n} \in \mathscr{F}_{0}$, is a Cauchy's sequence in the uniform norm. For this, given finite volume semigroups $P_{t}^{(l)} \equiv e^{t \mathbf{L}_{l}} \equiv P_{t}^{\mathbf{J}, \Lambda_{m_{l}}},(l=1,2)$, corresponding to a given configuration $\mathbf{J}$ and the cubes $\Lambda^{(l)} \equiv \Lambda_{m_{l}},(l=1,2)$, satisfying $\Lambda^{(1)} \subset \Lambda^{(2)}$, we need to estimate the uniform norm of the difference $P_{t}^{(2)} f-P_{t}^{(1)} f$. It will be sufficient to do that in the case when $\Lambda^{(1)}$ and $\Lambda^{(2)}$ differ by one point. Then we have

$$
P_{t}^{(2)} f-P_{t}^{(1)} f=\int_{0}^{t} d s P_{t-s}^{(1)}\left(\mathbf{L}_{2}-\mathbf{L}_{1}\right) P_{s}^{(2)} f .
$$

If $\Lambda^{(2)}=\Lambda^{(1)} \cup\{j\}$ we have

$$
\left(\mathbf{L}_{2}-\mathbf{L}_{1}\right) P_{s}^{(2)} f=\nabla_{j} H_{j, 2} \cdot \nabla_{j} P_{s}^{(2)} f,
$$

where we have set $H_{j, 2} \equiv H_{j, \Lambda^{(2)}}$. Since

$$
\left\|\left(\mathbf{L}_{2}-\mathbf{L}_{1}\right) P_{s}^{(2)} f\right\| \leqq\left\|\nabla_{j} H_{j, 2}\right\| \cdot\left\|\nabla_{j} P_{s}^{(2)} f\right\| \leqq b_{j}\left\|\nabla_{j} P_{s}^{(2)} f\right\|
$$

where

we get

$$
b_{j} \equiv\left(\sum_{\substack{X \in \mathscr{F} \\ X \ni j}}\left\|\nabla_{j} \Phi_{X}\right\|\right),
$$

$$
\left\|P_{t}^{(2)} f-P_{t}^{(1)} f\right\|_{u} \leqq b_{j} \int_{0}^{t} d s\left\|\nabla_{j} P_{s}^{(2)} f\right\|
$$

with $\{j\} \equiv \Lambda^{(2)} \backslash \Lambda^{(1)}$. Thus to complete this estimate we need to study $\nabla_{j} P_{s}^{(2)} f$ for $j \in \Lambda^{(2)}$. We note that

$$
\begin{gathered}
\left|\nabla_{j} P_{t}^{(2)} f\right|^{2}=P_{t}^{(2)}\left|\nabla_{j} f\right|^{2}+\int_{0}^{t} d s P_{t-s}^{(2)}\left(-\mathbf{L}_{2}\left|\nabla_{j} P_{s}^{(2)} f\right|^{2}+2\left(\nabla_{j} \mathbf{L}_{2} P_{s}^{(2)} f, \nabla_{j} P_{s}^{(2)} f\right)\right) \\
\leqq P_{t}^{(2)}\left|\nabla_{j} f\right|^{2}+\int_{0}^{t} d s P_{t-s}^{(2)} 2 \mid \operatorname{Ric}\left(\nabla_{j} P_{s}^{(2)} f, \nabla_{j} P_{s}^{(2)} f\right) \\
\quad+\sum_{k \in \Lambda^{(2)}} \operatorname{Hess}_{j k}^{(2)}\left(\nabla_{j} P_{s}^{(2)} f, \nabla_{k} P_{s}^{(2)} f\right) \mid
\end{gathered}
$$


where $\operatorname{Hess}_{j k}^{(2)} \equiv \operatorname{Hess}_{j k}^{H_{\Lambda^{(2)}}}$. Since under our assumptions we have

$$
\left.\left|\operatorname{Ric}\left(\nabla_{j} P_{s}^{(2)} f, \nabla_{j} P_{s}^{(2)} f\right)\right| \leqq \bar{\kappa} \mid \nabla_{j} P_{s}^{(2)} f\right)\left.\right|^{2}
$$

with some $\bar{\kappa} \in \mathbb{R}^{+}$and

$$
\left|\operatorname{Hess}_{j k}^{(2)}\left(\nabla_{j} P_{s}^{(2)} f, \nabla_{k} P_{s}^{(2)} f\right)\right| \leqq \sum_{k}\left(\sum_{\substack{X \in \mathscr{F} \\ X \ni j, k}}\left\|\nabla_{j} \nabla_{k} \Phi_{X}\right\|\right)\left|\nabla_{j} P_{s}^{(2)} f\right| \cdot\left|\nabla_{k} P_{s}^{(2)} f\right|
$$

combining with (10) we obtain

$$
\left\|\nabla_{j} P_{t}^{(2)} f\right\|^{2} \leqq\left\|\nabla_{j} f\right\|^{2}+\int_{0}^{t} d s \sum_{k: d(j, k) \leqq R} G_{j k}\left\|\nabla_{j} P_{s}^{(2)} f\right\| \cdot\left\|\nabla_{k} P_{s}^{(2)} f\right\|
$$

with a matrix

$$
G_{j k} \equiv G_{j k}(\mathbf{J}) \equiv 2 \bar{\kappa} \delta_{j k}+2 \sum_{\substack{X \in \mathscr{F} \\ X \ni j, k}}\left\|\nabla_{j} \nabla_{k} \Phi_{X}\right\|
$$

Whence we get

$$
\left\|\nabla_{j} P_{t}^{(2)} f\right\| \leqq\left\|\nabla_{j} f\right\|+\int_{0}^{t} d s \sum_{k: d(j, k) \leqq R} G_{j k}\left\|\nabla_{k} P_{s}^{(2)} f\right\| .
$$

Iterating this inequality and taking into account that $\nabla_{k} f=0$ if $k \notin \Lambda(f)$, we arrive at the following bound:

$$
\left\|\nabla_{j} P_{t}^{(2)} f\right\| \leqq \sum_{i \in \Lambda(f)} \sum_{\substack{n \in \mathbb{N} \\ n \geqq d(j, i)}} \frac{t^{n}}{n !} \sum_{\substack{k_{0}, \ldots, k_{n} \in \Lambda^{(2)} \\ d\left(k_{l}, k_{l+1}\right) \leqq R \\ k_{0}=i, k_{n}=j}} \prod_{l=1}^{n} G_{k_{l-1}, k_{l}}\left\|\nabla_{i} f\right\| .
$$

To proceed further, let us assume that there is an increasing positive function $\varrho$ such that

$$
G_{k_{l-1}, k_{l}} \leqq \varrho\left(d\left(k_{l}, 0\right)\right) \leqq \varrho(R l+d(i, 0)),
$$

where we have taken into account that starting from a point $i$ at each step we can move at most at the distance $R$. Using this assumption, we see that for $j \in \Lambda^{(2)} \backslash \Lambda^{(1)}$ we have

$$
\prod_{l=1}^{n} G_{k_{l-1}, k_{l}} \leqq\left(\prod_{l=1}^{[d(j, i) / R]} \varrho(R l+d(i, 0))\right) \varrho(d(j, 0))^{n-[d(j, i) / R]}
$$

Since the maximal number of walks from the origin to the point $j$ does not exceed $(2 R)^{d n}$, we obtain the following estimate when $j \in \Lambda^{(2)} \backslash \Lambda^{(1)}$ :

$$
\left\|\nabla_{j} P_{t}^{(2)} f\right\| \leqq \sum_{i \in \Lambda(f)} \frac{\left((2 R)^{d} t\right)^{d(j, i)}}{d(j, i) !}\left(\prod_{l=1}^{[d(j, i) / R]} \varrho(R l+d(i, 0))\right) e^{(2 R)^{d} t \varrho(d(j, 0))}\left\|\nabla_{i} f\right\| .
$$


Now using this and (9) we arrive at the following bound:

$$
\begin{aligned}
& \left\|P_{t}^{(2)} f-P_{t}^{(1)} f\right\|_{u} \\
& \quad \leqq b_{j} \cdot t \cdot \max _{i \in \Lambda(f)}\left(\frac{\left((2 R)^{d} t\right)^{d(j, i)}}{d(j, i) !}\left(\prod_{l=1}^{[d(j, i) / R]} \varrho(R l+d(i, 0))\right) e^{(2 R)^{d} t \varrho(d(j, 0))}\right)\|\| f\|\| .
\end{aligned}
$$

From (20), by induction, for arbitrary sets $\Lambda_{m}, \Lambda_{n} \in \mathscr{F}_{0}$ we obtain

$$
\begin{aligned}
\left\|P_{t}^{\left(\Lambda_{m}\right)} f-P_{t}^{\left(\Lambda_{n}\right)} f\right\|_{u}= & \sum_{j \in \Lambda_{m} \backslash \Lambda_{n}} b_{j} \cdot t \cdot \max _{i \in \Lambda(f)}\left(\frac{\left((2 R)^{d} t\right)^{d(j, i)}}{d(j, i) !}\right. \\
& \left.\times\left(\prod_{l=1}^{[d(j, i) / R]} \varrho(R l+d(i, 0))\right) e^{(2 R)^{d} t \varrho(d(j, 0))}\right)\|f\| \| .
\end{aligned}
$$

To complete our estimates and the proof of existence we need the following probabilistic lemma.

\section{Lemma 2.2. Suppose}

$$
\sup _{X \in \mathscr{F}, i, j \in \mathbb{Z}^{d}} \mathbb{E}\left\|\nabla_{i} \nabla_{j} \Phi_{X}\right\|^{K}<\infty
$$

with some $K>d$. Then there is $\delta \in(0,1)$ such that

$$
\sup _{\substack{i, j: d(i, 0), d(j, i) \leqq R \\ X: \operatorname{diam}(X) \leqq R}}\left\|\nabla_{i+k} \nabla_{j+k} \Phi_{X+k}\right\| \leqq C_{2}(\mathbf{J})(1+d(k, 0))^{\delta},
$$

and therefore

$$
\sup _{\substack{i: d(i, X) \leqq R \\ X: \operatorname{diam}(X) \leqq R}}\left\|\nabla_{i+k} \Phi_{X+k}\right\| \leqq C_{1}(\mathbf{J})(1+d(k, 0))^{\delta}
$$

with some constants $C_{1}(\mathbf{J})$ and $C_{2}(\mathbf{J})$ dependent only on $\mathbf{J} \in \mathbf{J}$.

Proof of Lemma 2.2. By simple considerations involving the Tschebyshev inequality, we see that for any $C \in(0, \infty)$ we have

$$
\begin{aligned}
& \mathbb{E}\left\{\exists k \in \mathbb{Z}^{d} \sup _{\substack{i, j: d(i, 0), d(j, i) \leqq R \\
X: \operatorname{diam}(X) \leqq R}}\left\|\nabla_{i+k} \nabla_{j+k} \Phi_{X+k}\right\| \geqq C(1+d(k, 0))^{\delta}\right\} \\
& \leqq \frac{2 R^{d} 2^{R^{d}}}{C^{K}} \sup _{i, j, X} \mathbb{E}\left\|\nabla_{l} \nabla_{j} \Phi_{X}\right\|^{K} \sum_{k \in \mathbb{Z}^{d}}(1+d(k, 0))^{-K \delta},
\end{aligned}
$$

the series on the right-hand side of (25) being convergent provided that $K \delta>d$. Since the positive constant $C$ was arbitrary, we conclude that

$$
\left\|\nabla_{i+k} \nabla_{j+k} \Phi_{X+k}\right\| \leqq C(\mathbf{J})(1+d(k, 0))^{\delta} \quad \text { E-a.e } .
$$


for every $k \in \mathbb{Z}^{d}$ and $i \in \mathbb{Z}^{d}, d(i, k) \leqq R$, with some constant $C(\mathbf{J}) \in(0, \infty)$. From this the property (23) easily follows. As $\mathbf{M}$ is compact, the inequality (24) is a consequence of (23). This ends the proof of Lemma 2.2.

Using the definition of $G_{i j}$ given in (17) we see that we need to choose the majorizing function $\varrho$ in the form

$$
\varrho(n) \equiv \varrho(\mathbf{J}, n) \equiv\left(2 \bar{\kappa}+C_{2}(\mathbf{J})\right)(1+n)^{\delta} \equiv C_{3}(\mathbf{J})(1+n)^{\delta} .
$$

Given this choice and setting $N=d(j, f)$, we see that

$$
\begin{aligned}
& t \cdot \max _{i \in \Lambda(f)}\left(\frac{\left((2 R)^{d} t\right)^{d(j, i)}}{d(j, i) !}\left(\prod_{l=1}^{[d(j, i) / R]} \varrho(R l+d(i, 0))\right) e^{(2 R)^{d} t \varrho(d(j, 0))}\right) \\
& \quad \leqq C_{4} t^{1+\operatorname{diam} \Lambda(f)} \frac{\left(C_{5} t\right)^{N}}{N !}\left(\prod_{l=1}^{[N / R]} \varrho(R l)\right) \exp \left\{(2 R)^{d} t \varrho\left(N+C_{6}\right)\right\} \\
& \quad \leqq C(\mathbf{J}, \Lambda(f)) \exp (F(N, t))
\end{aligned}
$$

with some positive constants $C_{4} \equiv C_{4}(\mathbf{J}, \Lambda(f))$ and $C_{5} \equiv C_{5}(\mathbf{J}, \Lambda(f))$ and $C_{6} \equiv$ $\operatorname{diam}(\Lambda(f))+d(f, 0)$, and

$$
F(N, t) \leqq N\left(\log t+C_{7}\right)+C_{8} t N^{\delta}+C_{9}(\log t+\log N)-(1-\delta) N \log N
$$

with some positive constants $C_{7} \equiv C_{7}(\mathbf{J}, \Lambda(f)), \quad C_{8} \equiv C_{8}(\mathbf{J}, \Lambda(f))$ and $C_{9} \equiv$ $C_{9}(\Lambda(f))$.

As a consequence, for any $L \geqq \bar{L}$, with some large constant $\bar{L} \equiv \bar{L}(\Lambda(f), \delta)$, and all $N \geqq L$, we have

$$
F(N, t) \leqq-A t-N
$$

provided that

$$
C t \leqq L^{1-\delta}
$$

with some sufficiently large constant $C \equiv C(\mathbf{J}, A)$. From (21) and (24) it is now clear that for every $\Lambda_{m}$ and $\Lambda_{n}$, with $\Lambda_{m} \subseteq \Lambda_{n}$ and $d\left(f, \Lambda_{m}^{c}\right) \geqq \bar{L}$ for some sufficiently large constant $\bar{L}$, we have with some constant $D(\mathbf{J})$

$$
\left\|P_{t}^{\left(\Lambda_{m}\right)} f-P_{t}^{\left(\Lambda_{n}\right)} f\right\|_{u} \leqq e^{-A t} D(\mathbf{J})\|\| f\|\| .
$$

Thus the sequence $\left\{P_{t}^{\left(\Lambda_{n}\right)}\right\}_{n \in \mathbb{N}}$ converges on a dense set of local $\mathscr{C}^{1}$ functions uniformly on compact intervals to a Markov semigroup $P_{t}$. The semigroup $P_{t}$ satisfies also the desired approximation property (3) with time growing with the size of $\Lambda_{n}$ in a sublinear way $(1 / C)\left(d\left(f, \Lambda^{c}\right)\right)^{1-\delta}$, for some $\delta \in(0,1)$, provided the distribution $\mathbb{E}$ of randomness satisfies the appropriate moment condition. This ends the proof of Theorem 2.1.

Remark. If the moment condition of Lemma 2.2 would be replaced by a finite expectation of the exponential of the corresponding quantity, we could take $\delta=0$, i.e. in this case we would have the same approximation property as in nonrandom case.

By similar arguments one can show in the discrete case the following result. 
Theorem 2.3. Suppose $\Phi \equiv\left\{\Phi_{X}\right\}_{X \in \mathscr{F}}$ is a (smooth) interaction of finite range $R$ and there is a $K>d$ such that

$$
\sup _{X \in \mathscr{F}, i, j \in \mathbb{Z}^{d}} \mathbb{E}\left\|\mathbf{a}_{X+i}\right\|^{K}<\infty
$$

Then the limit

$$
\mathscr{P}_{t}^{\mathbf{J}} f \equiv \lim _{\mathscr{F}_{0}} \mathscr{P}_{t}^{\mathbf{J}, \Lambda} f
$$

exists $\mathbb{E}$-a.e. Moreover for any cube $\Lambda$, any $A \in \mathbb{R}^{+}$and any local function $f \in \mathbb{B}_{0}$ we have

$$
\left\|\mathscr{P}_{t}^{\mathbf{J}} f-\mathscr{P}_{t}^{\mathbf{J}, \Lambda} f\right\|_{u} \leqq e^{-A t} D(\mathbf{J})\|\| f\|\|,
$$

provided that $d\left(f, \Lambda^{c}\right) \geqq \bar{L}$ for some sufficiently large constant $\bar{L} \equiv \bar{L}(\Lambda(f), R, \mathbb{E})$ and

$$
d\left(f, \Lambda^{c}\right)^{1-\delta} \geqq C t
$$

with a constant $C \in \mathbb{R}^{+}$dependent on $\mathbf{J}, \Lambda(f)$ and the choice of $A$, and with some $\delta \in(0,1)$ such that $K \delta>d$.

\section{No Spectral Gap Property}

We say that a generator $\mathbf{L}$, as a selfadjoint operator in $\mathbb{L}_{2}(\mu)$, has a spectral gap iff

$$
0<\operatorname{gap}_{2}(\mathbf{L}) \equiv \operatorname{gap}_{L_{2}(\mu)}(\mathbf{L}) \equiv \inf _{f \in \mathscr{D}(\mathbf{L}), f \perp \mathbb{1}} \frac{\mu(f(-\mathbf{L} f))}{\mu(f, f)} .
$$

In this section we show the absence of the spectral gap property, with probability one, for some spin systems with random interactions at high temperatures. A similar property has been first proven in [39] in the case of the discrete Ising model on $\mathbb{Z}^{d}$ with nearest neighbour random couplings taking large values with nonzero probability. In the discrete case the proof given in [39] was strongly based on the optimal volume dependence of the spectral gap obtained in [37] for an Ising ferromagnetic model with free boundary conditions. As there is no reason to expect that such behaviour of the spectral gap will be true in the general continuous case, (in particular in dimension two if the interaction is invariant with respect to a continuous group of symmetries), we need to modify our previous strategy. For this we observe the following simple fact.

Lemma 3.1. For any Gibbs measure $\mu$ for a local specification $\mathscr{E} \equiv\left\{\mu_{\Lambda}^{\omega}\right\}_{\Lambda \in \mathscr{F}, \omega \in \Omega}$ we have

$$
\inf _{f \in \mathscr{D}(\mathbf{L}), f \perp \mathbb{1}} \frac{\mu(f(-\mathbf{L} f))}{\mu(f, f)} \leqq \inf _{\Lambda \in \mathscr{F}}\left(\inf _{\substack{f \in \mathscr{D}(\mathbf{L}), f \perp \mathbb{1} \\ \Lambda(f) \subset \Lambda}}\left\|\frac{\mu_{\Lambda}^{\omega}(f(-\mathbf{L} f))}{\mu_{\Lambda}^{\omega}(f, f)}\right\|_{u}\right) .
$$


Proof. For $f \in \mathscr{D}(\mathbf{L})$ with $\Lambda(f) \subset \Lambda$ and $f \perp \mathbb{l}$, we have

$$
\begin{aligned}
\frac{\mu(f(-\mathbf{L} f))}{\mu(f, f)} & =\frac{\mu\left(\mu_{\Lambda}^{\omega}(f(-\mathbf{L} f))\right)}{\mu(f, f)} \leqq\left\|\frac{\mu_{\Lambda}^{\omega}(f(-\mathbf{L} f))}{\mu_{\Lambda}^{\omega}(f, f)}\right\|_{u} \frac{\mu\left(\mu_{\Lambda}^{\omega}(f, f)\right)}{\mu(f, f)} \\
& \leqq\left\|\frac{\mu_{\Lambda}^{\omega}(f(-\mathbf{L} f))}{\mu_{\Lambda}^{\omega}(f, f)}\right\|_{u} .
\end{aligned}
$$

This implies the lemma.

For our problem it is convenient to consider local functions of the following form:

$$
f \equiv \sum_{j} h_{j} \psi_{j}
$$

where $\psi_{j} \equiv T_{j} \psi$, with some local function $\psi \in \mathscr{D}(\mathbf{L}), \psi \neq$ const and a collection of real numbers $h_{j}, j \in \mathbb{Z}^{d}$. In some applications it will be convenient to consider $\psi$ as a vector valued function. Then one will need also to adjust the interpretation of squares.

Then, taking into account also the local structure of our Markov generator, we get the following convenient upper bound for the spectral gap.

\section{Lemma 3.2.}

$$
\operatorname{gap}_{2}(\mathbf{L}) \leqq \sup _{\omega} \frac{\sum_{j \in \Lambda} h_{j}^{2} \mu_{\Lambda}^{\omega}\left|\nabla_{j} \psi_{j}\right|^{2}}{\sum_{i, j \in \Lambda} h_{i} h_{j} \mu_{\Lambda}^{\omega}\left(\psi_{i}, \psi_{j}\right)} .
$$

In the discrete case for single spin flip dynamics we get

$$
\operatorname{gap}_{2}(\mathscr{L}) \leqq \sup _{\omega} \frac{\sum_{i: X+i \subset \Lambda}\left(\sum_{j, k \in X+i} h_{j} h_{k} \mu_{\Lambda}^{\omega}\left(\mu_{X+i}\left(\psi_{j}, \psi_{k}\right)\right)\right)}{\sum_{j, k} h_{j} h_{k} \mu_{\Lambda}^{\omega}\left(\psi_{j}, \psi_{k}\right)}
$$

which in the particular case of the single spin flip dynamics gives

$$
\operatorname{gap}_{2}(\mathscr{L}) \leqq \sup _{\omega} \frac{\sum_{j \in \Lambda} h_{j}^{2} \mu_{\Lambda}^{\omega}\left(\mu_{j}\left(\psi_{j}, \psi_{j}\right)\right)}{\sum_{i, j \in \Lambda} h_{i} h_{j} \mu_{\Lambda}^{\omega}\left(\psi_{i}, \psi_{j}\right)}
$$

Consider now a spin system with random interaction $\Phi \equiv\left\{\Phi_{X}\right\}_{X \in \mathscr{F}}$ of finite range $R$, on a lattice $\mathbb{Z}^{d}$. Let us recall that the random variable $\Phi_{X}(\cdot, \omega), X \in \mathscr{F}$, $\omega \in \Omega$ are mutually independent. For any $j \in \mathbb{Z}^{d}$ we define a translation invariant interaction

$$
\tilde{\Phi}^{(j)} \equiv\left\{\tilde{\Phi}_{X+k}^{(j)}(\omega) \equiv \Phi_{X}\left(\mathbf{J}, T_{k} \omega\right): k \in \mathbb{Z}^{d}, X \ni j\right\} .
$$

We assume that there is a set $\mathcal{O}$ of Gibbsian interactions for which the corresponding theory satisfies the Dobrushin-Shlosman uniqueness condition [9] and we have

$$
\forall j \in \mathbb{Z}^{d} \quad \mathbb{E}\left\{\tilde{\Phi}^{(j)} \in \mathcal{O}\right\} \geqq p_{0}
$$

with $p_{0} \in(1 / 2,1)$ a positive number to be chosen later. In order to avoid the necessity of considering all the boundary conditions when using Lemma 3.1, we observe the following simple fact $([1,28])$. 
Lemma 3.3. Suppose for $f \in \mathscr{D}(\mathbf{L})$ we have $d\left(f, \Lambda^{c}\right)>R$ and $\tilde{\Phi}^{(j)} \in \mathcal{O}$ for all $j \in \Lambda \backslash \Lambda(f)$. Then

$$
\left|\mu_{\Lambda}^{\omega}(f(-\mathbf{L} f))-\mu_{\Lambda}^{B D}(f(-\mathbf{L} f))\right| \leqq C_{1}(f)|\partial \Lambda| e^{-M d\left(f, \Lambda^{c}\right)}
$$

and

$$
\left|\mu_{\Lambda}^{\omega}(f, f)-\mu_{\Lambda}^{B D}(f, f)\right| \leqq C_{2}(f)|\partial \Lambda| e^{-M d\left(f, \Lambda^{c}\right)}
$$

with some constant $M>0$ independent of $\Lambda, f$ and $d\left(f, \Lambda^{c}\right)$, and some constants $C_{1}(f)$ and $C_{2}(f)$ dependent only on the function $f$ (and $\left.\mathcal{O}\right)$; here $\mu_{\Lambda}^{B D}$ denotes a finite volume measure with free, periodic or given by some other configuration, boundary conditions.

Remark. A similar result remains true in the discrete case.

The final preparatory remark is the following probabilistic lemma.

Lemma 3.4. Suppose for any $j \in \mathbb{Z}^{d}$ and some set $\mathcal{O}_{1} \supset \mathcal{O}$, we have

$$
\forall j \in \mathbb{Z}^{d} \quad 0<p_{1}<\mathbb{E}\left\{\tilde{\Phi}^{(j)} \notin \mathcal{O}_{1}\right\}
$$

with some $p_{1} \in(0,1)$. Then for any number $C \in(0, \infty)$, with $\mathbb{E}$ probability one for every configuration $\mathbf{J} \in \mathbb{J}$ there exists an infinite sequence of disjoint cubes $\Lambda_{n}$, $n \in \mathbb{N}$, with $\operatorname{diam}\left(\Lambda_{n}\right) \rightarrow_{n \rightarrow \infty} \infty$, for which we have

$$
\forall j \text { such that } R \leqq d\left(j, \Lambda_{n}\right) \leqq C \log \left|\Lambda_{n}\right|, \quad \tilde{\Phi}^{(j)} \in \mathcal{O}
$$

and

$$
\forall j \in \Lambda_{n}, \quad \tilde{\Phi}^{(j)} \notin \mathcal{O}_{1} .
$$

Proof. Let us set

$$
\partial_{C \log } \Lambda \equiv\left\{j \in \Lambda^{c}: d(j, \Lambda) \leqq C \log |\Lambda|\right\} .
$$

We need only to observe that the probability of an event $\mathscr{A}_{n}$ described by (47) and (48) satisfies

$$
\mathbb{E}\left\{\mathscr{A}_{n}\right\} \geqq p_{1}^{\left|\Lambda_{n}\right|} p_{0}^{\left|\partial_{C \log } \Lambda_{n}\right|} \geqq\left(p_{0} p_{1}\right)^{\left|\Lambda_{n}\right|}
$$

Thus if

$$
\left|\Lambda_{n}\right|=\left(\left|\log \left(p_{0} p_{1}\right)\right|\right)^{-1} \log n
$$

then

$$
\sum_{n} \mathbb{E}\left\{\mathscr{A}_{n}\right\}=\infty
$$

and the conclusion of the lemma follows by the Borel-Cantelli lemma.

As an interesting example we would like to consider the two-dimensional plane rotator model with random nearest neighbour interaction, i.e. a model on $\mathbb{Z}^{2}$ with single spin space $\mathbf{M} \equiv S^{1}$ and the interaction given by

$$
\Phi_{X}= \begin{cases}J_{i j} \cos \left(\varphi_{i}-\varphi_{j}\right) & \text { if } X=\{i j\} \\ 0 & \text { otherwise }\end{cases}
$$


with $J_{i j}$ being i.i.d. random variables such that

$$
\mathbb{E}\left\{J_{i j} \in\left[0, J_{0}\right)\right\} \equiv p_{0}>0,
$$

where $J_{0} \in(0, \infty)$ is a value of the coupling for which the corresponding infinite system is in the Dobrushin-Shlosman uniqueness region, and

$$
\mathbb{E}\left\{J_{c}+1>J_{i j}>J_{c}\right\} \equiv p_{1}>0,
$$

where $J_{c}>0$ is a coupling for the nonrandom rotator model in the region of the Kosterlitz-Thouless phase considered in [19].

We will use the lemmata proven above to show the following result.

Theorem 3.5. In the two-dimensional plane rotator model with random nearest neighbours couplings satisfying (53) and (54), we have

$$
\operatorname{gap}_{2}(\mathbf{L})=0, \quad \mathbb{E}-\text { a.e } .
$$

Proof. According to Lemma 3.4, for any number $C>0$, with $\mathbb{E}$-probability one there is an infinite sequence of boxes $\Lambda_{n}$ with $\left|\Lambda_{n}\right|=\left(\left|\log p_{1} p_{2}\right|\right)^{-1} \log n$, with $p_{1}$ given by (54), and such that

$$
\forall\{i, j\} \subset \Lambda_{n} \quad J_{i j} \in\left(J_{c}, J_{c}+1\right)
$$

and

$$
\forall\{i, j\} \subset \partial_{C \log \Lambda_{n}} \quad J_{i j} \in\left[0,+J_{0}\right) .
$$

Now it is convenient to choose $\psi$ to be a vector $\sigma_{0} \equiv\left(\sigma_{0}^{(1)}, \sigma_{0}^{(2)}\right) \equiv$ $\left(\cos \varphi_{0}, \sin \varphi_{0}\right)$. In this representation we have

$$
\left|\nabla_{j} \sigma_{j}\right|^{2}=\left|\frac{d}{d \varphi}\left(\cos \varphi_{j}, \sin \varphi_{j}\right)\right|^{2}=1
$$

We will take $h_{j}=1$ for $j \in \Lambda_{n}$ and zero otherwise. Using Lemma 3.3 and (40), with some large constant $C \in(0, \infty)$, we obtain the following bound for the spectral gap of the generator $\mathbf{L}_{\mathbf{J}}$ in $\mathbb{L}_{2}\left(\mu_{\mathbf{J}}\right)$,

$$
\operatorname{gap}_{2}\left(\mathbf{L}_{\mathbf{J}}\right) \leqq \sup _{\omega}\left(\frac{1}{\left|\Lambda_{n}\right|} \sum_{j, k \in \Lambda_{n}} \mu_{\mathbf{J}, \Lambda_{n} \cup \partial_{C \log } \Lambda_{n}}\left(\cos \left(\varphi_{j}-\varphi_{k}\right)\right)+o\left(\left|\Lambda_{n}\right|^{-1}\right)\right)^{-1}
$$

where $\mu_{\mathbf{J}, \Lambda_{n} \cup \partial_{C \log } \Lambda_{n}}$ denotes the finite volume Gibbs measure with free boundary conditions, and

$$
o\left(\left|\Lambda_{n}\right|^{-1}\right) \equiv D\left|\Lambda_{n}\right|^{2} \cdot e^{-M C \log \left|\Lambda_{n}\right|}
$$

with some positive constant $D$ independent of $\Lambda_{n}$ and the choice of $C$.

Since by our choice of $\Lambda_{n}$ 's all the couplings are ferromagnetic, we can use correlation inequalities of Ginibre, (see e.g. [12] and references given there), to

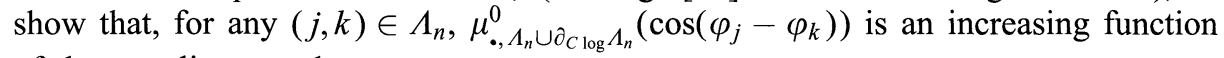
of the couplings so that:

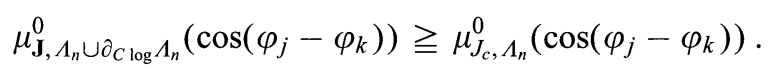


The couplings in the measure on the right-hand side of (60) are all equal to $J_{c}, J_{c}$ being a sufficiently large positive constant, so that the corresponding infinite system is in the region of the Kosterlitz-Thouless phase. Therefore we can use the estimate of [19]

$$
\mu_{J_{c}, \Lambda_{n}}^{0}\left(\cos \left(\varphi_{j}-\varphi_{k}\right)\right) \geqq \frac{\text { const }}{(1+d(j, k))^{a / J_{c}}}
$$

with some positive constants $a$ and const independent of $j, k \in \Lambda_{n}$ and $J_{c}$. Thence we conclude that for large boxes $\Lambda_{n}$ we have

$$
\operatorname{gap}_{2}\left(\mathbf{L}_{\mathbf{J}}\right) \leqq\left|\Lambda_{n}\right|^{-\left(1-a / 2 J_{c}\right)} .
$$

Clearly for sufficiently large $J_{c}$ we can have $a / 2 J_{c}<1$. Since $\left|\Lambda_{n}\right|$ diverges to infinity with $n$, this implies that the spectral gap of $\mathbf{L}_{\mathbf{J}}$ equals zero, $\mathbb{E}$-a.e.

The proof allows an extension to many other cases of random systems. We would like to mention here two simple systems on $\mathbb{Z}^{2}$ with discrete spins related to the ferromagnetic Ising model at the critical point and to the $Z_{n}$ model for which the Kosterlitz-Thouless phase transition has been shown in [19]. We have the following result.

Theorem 3.6. Suppose $\mathbf{M}=\{-1,+1\}$ or $\mathbf{M}=\{0, \ldots, n-1\}$ with some $n \in \mathbb{N}$, $n>1$. Let the interaction be given by

$$
\Phi_{X}=\left\{\begin{array}{ll}
-J_{i j} \sigma_{i} \sigma_{j} & \text { if } X=\{i j\} \\
0 & \text { otherwise }
\end{array},\right.
$$

or as in the plane rotator model with $\varphi \in\{2 \pi k / n: k=0, \ldots, n-1\}$, in both cases with $J_{i j}$ being i.i.d. random variables such that

$$
\mathbb{E}\left\{J_{i j} \in\left[0, J_{0}\right)\right\} \equiv p_{0}>0
$$

and

$$
\mathbb{E}\left\{J_{i j}=J_{c}\right\} \equiv p_{1}>0,
$$

where $J_{c}>0$ is a coupling for the nonrandom Ising and $Z_{n}$ model at the critical point and in the region of the Kosterlitz-Thouless phase, respectively. Then

$$
\operatorname{gap}_{2}\left(\mathscr{L}_{\mathbf{J}}^{(0)}\right)=0, \quad \mathbb{E} \text {-a.e. }
$$

where $\mathscr{L}_{\mathbf{J}}^{(0)}$ is the single spin flip generator.

\section{Remark.}

- In the proof for the case of $Z_{n}$ models one has to use the correlation inequalities of [30] and [19].

- Let us recall that for the ferromagnetic Ising model with nearest neighbours interactions, one has a spectral gap up to the critical point, [28]. 


\section{Decay to Equilibrium in Disordered Spin Systems on $\mathbb{Z}^{2}$}

In this section we consider (discrete and continuous) spin systems on the two dimensional lattice. We show that under some restrictions on the distribution of random interaction, (which are typically satisfied in the random spin systems at high temperatures), the corresponding stochastic dynamics has with probability one a stretched exponential upper bound for the decay to equilibrium of local observables. By this we strengthen the results of [20] concerning the upper bound of decay to equilibrium obtained for discrete spin systems. The method of [20] gives a decay for local observables faster than any algebraic, but much slower than the stretched exponential. It is based on some disagreement percolation technique and therefore essentially uses the fact that the single spin space is discrete. Our method is based on the use of the logarithmic Sobolev inequality and is free of this restriction.

In this section we assume that we are given a random interaction $\Phi \equiv\left\{\Phi_{X}\right\}_{X \in \mathscr{F}}$ of finite range $R>0$, for which a stochastic dynamics considered in Sect. 2, Theorem 2.3, exists, and which satisfies the following hypotheses.

(H1) For all $\xi \in \mathbb{R}$ we have

$$
\mathbb{E} \exp \left\{\xi\left\|\tilde{\Phi}^{(j)}(\mathbf{J}, \cdot)\right\|_{1}\right\}<\infty,
$$

where

$$
\left\|\tilde{\Phi}^{(j)}(\mathbf{J}, \cdot)\right\|_{1} \equiv \sum_{\substack{X \in \mathscr{F} \\ X \ni j}}\left\|\Phi_{X}(\mathbf{J}, \cdot)\right\|_{u},
$$

and in the continuous case

$$
\sup _{k, j} \mathbb{E} \exp \left\{\xi\left\|\nabla_{k} \nabla_{j} \tilde{\Phi}^{(j)}(\mathbf{J}, \cdot)\right\|_{1}\right\}<\infty .
$$

(H2) There is a small constant $J_{0}>0$ (specified later), such that

$$
0<p_{1} \equiv \sup _{X} \mathbb{E}\left\{\left\|\Phi_{X}\right\|_{u}>J_{0}\right\}<p_{c}^{b}(2, R),
$$

where $p_{c}^{b}(2, R)$ is a clustering coefficient (see Kesten [25] and Lemma 5.4 below).

We consider the stochastic dynamics constructed in Sect. 3 with the following additional assumption in discrete case:

(HD) For all $\zeta \in \mathbb{R}$, we have

$$
\sup _{j} \mathbb{E} \exp \left\{\zeta\left\|\left(\mathbf{a}_{X+j}(\mathbf{J}, \cdot)\right)^{-1}\right\|_{1}\right\}<\infty .
$$

We remark that, as we have seen in Sect. 2, under the conditions $\mathbb{H} 1$ and $\mathbb{H D}$ we have with probability one the exponential approximation property for the infinite volume semigroup up to linear time in the size of the finite volume. The condition $\mathbb{H} 2$ assures that with probability one we have uniqueness and exponential cluster property for equilibrium measures.

In view of Sect. 3 one can have already an interesting situation if the random interaction is bounded, but can take sufficiently large values with nonzero probability. The assumptions given above allows us also to include the case when the interaction is unbounded, but has a moderate growth when we move to infinity.

In this section we prove the following result. 
Theorem 4.1. Suppose the conditions $\mathbb{H} 1-\mathbb{H} 2$ are satisfied with $p_{1} \in\left(0, \bar{p}_{1}\right)$ and $J_{0} \in\left(0, \bar{J}_{0}\left(p_{1}\right)\right)$ for some $\bar{p}_{1} \in\left(0, p_{c}^{b}(2, R)\right)$ and $\bar{J}_{0}\left(p_{1}\right)>0$ sufficiently small. Then in the continuous case there is $\theta \in(0,1)$ such that for any local function $f \in \mathscr{C}^{1}$, with $\mathbb{E}$ probability one, we have

$$
\left\|P_{t}^{\mathbf{J}} f-\mu_{\mathbf{J}}(f)\right\|_{u} \leqq C(\Lambda(f), \mathbf{J}) e^{-t^{\theta}}\|\| f\|\|
$$

for all $t \geqq T(\Lambda(f), \mathbf{J})$, with some positive random variables $C(\Lambda(f), \mathbf{J})$ and $T(\Lambda(f), \mathbf{J})$. Similarly in the discrete case, if also IHD holds with $\zeta \in\left(\zeta_{0}, \infty\right)$, for some sufficiently large $\zeta_{0}>0$, then we have

$$
\left\|\mathscr{P}_{t}^{\mathbf{J}} f-\mu_{\mathbf{J}}(f)\right\|_{u} \leqq C^{\prime}(\Lambda(f), \mathbf{J}) e^{-t^{\theta}}\|\| f\|\|
$$

for all $t \geqq T^{\prime}(\Lambda(f), \mathbf{J})$, with some finite positive random variables $C^{\prime}(\Lambda(f), \mathbf{J})$ and $T^{\prime}(\Lambda(f), \mathbf{J})$.

This result is derived as a consequence of the following interesting property:

Theorem 4.2. Suppose the conditions of Theorem 4.1 are satisfied. Then for any cube $\Lambda_{n} \equiv[-n,+n]^{2}$, for almost all $\mathbf{J} \in \mathbb{J}$, there exists a positive integer $N(\mathbf{J})$ such that, for any $n \geqq N(\mathbf{J})$,

$$
\mu_{\mathbf{J}, \Lambda_{n}}^{0} f^{2} \log f^{2} \leqq 2 c_{\mathbf{J}, \Lambda_{n}} \mu_{\mathbf{J}, \Lambda_{n}}^{0}\left(f\left(-\mathscr{L}_{\mathbf{J}, \Lambda_{n}} f\right)\right)+\mu_{\mathbf{J}, \Lambda_{n}}^{0} f^{2} \log \mu_{\mathbf{J}, \Lambda_{n}}^{0} f^{2}
$$

with

$$
\mathbf{c}_{\mathbf{J}, \Lambda_{n}} \leqq c(\mathbf{J}, X) n^{1-\vartheta}
$$

with some random variable $c(\mathbf{J}, X) \in(0, \infty)$ and $\vartheta \in(\theta, 1)$ both independent of $n$, boundary conditions and the function $f$. The similar result remains true for conditional measures $\mu_{\mathbf{J}, \Lambda}^{\omega}$ uniformly in $\omega$, as well as in the continuous case with the corresponding generator $\mathbf{L}_{\mathbf{J}, \Lambda}$.

Remark. As it will be clear later from our proof, to be able to bound the logarithmic Sobolev constant $\mathbf{c}_{\mathbf{J}, \Lambda_{n}}$, we need that, for a given $\eta \in(0,1)$, a finite $C(R)>0$ which only depends on $R$ and an $r_{0} \geqq \bar{r}_{0}$ where $\bar{r}_{0}$ only depends on $\left(p_{1}, \eta, R\right)$, $\bar{r}_{0} \rightarrow 0$ when $p_{1} \rightarrow 0$, we have:

$$
\frac{\eta}{R} r_{0} \log C(R)\left(e^{4 J_{0}}-1\right)+12 \inf _{s>0}\left(2^{5} r_{0} R \frac{1}{s} \log \mathbb{E}\left[\exp \left\{s\left\|\tilde{\Phi}^{(j)}\right\|_{1}\right\}\right]+\frac{3}{s}\right)<0 .
$$

Then, to get a growth of order $n^{1-\vartheta}$ with some positive constant $\vartheta$, we need that:

$$
12 \inf _{s>0}\left(2^{5} r_{0} R \frac{1}{s} \log \mathbb{E}\left[\exp \left\{s\left\|\tilde{\Phi}^{(j)}\right\|_{1}\right\}\right]+\frac{3}{s}\right)<1 .
$$

Now we will present the proof of Theorem 4.1, the main result of this section, assuming Theorem 4.2 (proof of which is contained in the next section). As both cases are very similar, we restrict ourselves to the discrete one, giving only 
a brief remark in the place where some modification for the continuous case is necessary.

Proof of Theorem 4.1. Let $f \in \mathscr{C}^{1}$ be a local function localised in a cube $\Lambda_{n_{0}} \equiv\left[-n_{0},+n_{0}\right]^{2}$. Let $\mathbb{J}_{0} \subset \mathbb{J}$ be a set of $\mathbb{E}$-measure one such that for every $\mathbf{J} \in \mathbb{J}_{0}$ Theorem 2.3 and Theorem 4.2 are true. Then taking $\Lambda_{n} \equiv[-n,+n]^{2}$ with $n \geqq n_{0} \in \mathbb{N}$ sufficiently large and $[C t]=n-n_{0}$, so that Theorem 2.3 is true, we get

$$
\begin{aligned}
\left|\mathscr{P}_{t}^{\mathbf{J}, X} f(\omega)-\mu_{\mathbf{J}} f\right| & \leqq\left|\mathscr{P}_{t}^{\mathbf{J}, X, \Lambda_{n}} f(\omega)-\mu_{\mathbf{J}, \Lambda_{n}}^{0} f\right|+\left\|\mathscr{P}_{t}^{\mathbf{J}, X} f-\mathscr{P}_{t}^{\mathbf{J}, X, \Lambda_{n}} f\right\|_{u}+\left|\mu_{\mathbf{J}} f-\mu_{\mathbf{J}, \Lambda_{n}}^{0} f\right| \\
& \leqq\left|\mathscr{P}_{t}^{\mathbf{J}, X, \Lambda_{n}} f(\omega)-\mu_{\mathbf{J}, \Lambda_{n}}^{0} f\right|+e^{-A t}|||f|\left\|\left|+e^{-M\left(n-n_{0}\right)}\right||| f|\||\right.
\end{aligned}
$$

for arbitrary chosen $A \in(0, \infty)$; the estimate of the last term with some constant $M>0$ independent of $\mathbf{J}$ and $f$ has been proven in [6] (see also [18, 7]). Therefore it is sufficient to bound the first term from the right-hand side. One can do that using Theorem 4.2 and the idea of [24] (see also [33-36 and 39]). We include a short proof for the readers' convenience. First we observe that for any $q \in(1, \infty)$ we have

$$
\begin{aligned}
\left|\mathscr{P}_{t}^{\mathbf{J}, X, \Lambda_{n}} f(\omega)-\mu_{\mathbf{J}, \Lambda_{n}}^{0} f\right| & =\left(\left|\mathscr{P}_{t}^{\mathbf{J}, X, \Lambda_{n}} f(\omega)-\mu_{\mathbf{J}, \Lambda_{n}}^{0} f\right|^{q}\right)^{\frac{1}{q}} \\
& \leqq e^{2\left\|H_{\mathbf{J}, \Lambda_{n}}\right\|_{u} / q}\left(\mu_{\mathbf{J}, \Lambda_{n}}^{0}\left|\mathscr{P}_{t}^{\mathbf{J}, X, \Lambda_{n}} f(\cdot)-\mu_{\mathbf{J}, \Lambda_{n}}^{0} f\right|^{q}\right)^{\frac{1}{q}} .
\end{aligned}
$$

(In the continuous case one needs to use the Hölder inequality and to run the semigroup for a unit time to get such an ultracontractivity estimate, [34].) We want to use the hypercontractivity for a small fraction $\delta \in(0,1)$ of our time, therefore we choose

$$
q \equiv 1+e^{2 \delta t / c_{n}}
$$

with $c_{n}=\mathbf{c}_{\mathbf{J}, \Lambda_{n}}$. Using the estimate on $c_{\mathbf{J}, \Lambda_{n}}$ given in Theorem 4.2 together with our assumption about the interaction, it is not difficult to see that with $\mathbb{E}$ probability one we have

$$
\lim _{t \rightarrow \infty} \frac{2\left\|H_{\mathbf{J}, \Lambda_{n}}\right\|_{u}}{1+e^{2 \delta t / c_{n}}}=1 .
$$

On the other hand, since Theorem 4.2 implies the hypercontractivity of the semigroup $\mathscr{P}_{s}^{\mathbf{J}, X, A_{n}}, s \geqq 0$, [22], we obtain

$$
\begin{aligned}
\left(\mu_{\mathbf{J}, \Lambda_{n}}^{0}\left|\mathscr{P}_{t}^{\mathbf{J}, X, \Lambda_{n}} f(\cdot)-\mu_{\mathbf{J}, \Lambda_{n}}^{0} f\right|^{q}\right)^{\frac{1}{q}} & \leqq\left(\mu_{\mathbf{J}, \Lambda_{n}}^{0}\left|\mathscr{P}_{(1-\delta) t}^{\mathbf{J}, X, \Lambda_{n}} f(\cdot)-\mu_{\mathbf{J}, \Lambda_{n}}^{0} f\right|^{2}\right)^{\frac{1}{2}} \\
& \leqq e^{-\frac{(1-\delta) t}{c_{n}}}\left(\mu_{\mathbf{J}, \Lambda_{n}}^{0}\left|f(\cdot)-\mu_{\mathbf{J}, \Lambda_{n}}^{0} f\right|^{2}\right)^{\frac{1}{2}} \\
& \leqq e^{-\frac{(1-\delta) t}{c_{n}}}|||f| \| \mid
\end{aligned}
$$

where we have used the fact that the logarithmic Sobolev inequality with a coefficient $c_{n}=c_{\mathbf{J}, \Lambda_{n}}$ implies a spectral gap at the bottom of the operator $-\mathscr{L}_{\mathbf{J}, \Lambda_{n}}^{X}$ of size not smaller than the inverse of that coefficient. Now application of the bound on the growth of $c_{n}$ of Theorem 4.2 together with our choice $[C t]=n-n_{0}$, (which is 
possible by our assumptions about the interaction), and (77)-(80), yields

$$
\begin{aligned}
\left|\mathscr{P}_{t}^{\mathbf{J}, X} f(\omega)-\mu_{\mathbf{J}} f\right| & \leqq\left(C_{0}(\Lambda(f), \mathbf{J}) e^{-\frac{(1-\delta) t}{c_{n}}}+e^{-A t}+e^{-M[C t]}\right)|\|f \mid\| \\
& \leqq\left(C_{0}(\Lambda(f), \mathbf{J}) e^{-\frac{(1-\delta) t^{\vartheta}}{c(\mathbf{J}, X) C^{1-\vartheta}}}+e^{-A t}+e^{-M[C t]}\right)|\|f\|| \\
& \leqq C(\Lambda(f), \mathbf{J}) e^{-t^{\theta}}|\|f \mid\|
\end{aligned}
$$

with some positive random variables $C_{0}(\Lambda(f), \mathbf{J})$ and $C(\Lambda(f), \mathbf{J})$. This ends the proof of Theorem 4.1 .

\section{Almost Sure Local Logarithmic Sobolev Inequality}

In this section we prove Theorem 4.2 for the case of conditional measures $\mu_{\Lambda}^{\omega}$. The case of free (or periodic) boundary conditions is similar. We will follow closely the lines of the proof given in [35], only underlying the main points which need to be changed.

Let $\left\{e_{l}\right\}_{0}^{3}$ be the enumeration of $\{0,1\}^{2}$ in which $l=\sum_{i=1}^{2} e_{l}^{i} 2^{2-i}$. Given $L \in \mathbb{N}$ (which will be specified later) an element of $\mathbb{N}$, we set:

$$
\begin{gathered}
\Gamma_{l}=\left\{(L+2 R) e_{l}+(2(L+2 R)) \mathbb{Z}^{2}\right\} \cap \Lambda, Y_{k}=\left\{k+[0,2(L+R)]^{2}\right\} \cap \Lambda, \\
\Lambda_{l}=\bigcup_{k \in \Gamma_{l}} Y_{k} .
\end{gathered}
$$

By construction the corresponding conditional expectation is a product measure

$$
\mu_{\Lambda_{l}}^{\omega} \equiv \bigotimes_{k \in \Gamma_{l}} \mu_{Y_{k}}^{\omega}
$$

and therefore using the product property for the logarithmic Sobolev coefficients, [22], we have

$$
\mathbf{c}_{L} \equiv \sup _{\omega \in \Omega} \max _{l=0, \ldots, 3}\left\{c\left(\mu_{\Lambda_{l}}^{\omega}\right)\right\}=\sup _{\omega \in \Omega} \max _{l=0, \ldots, 3}\left(\max _{k \in \Gamma_{l}}\left\{c\left(\mu_{Y_{k}}^{\omega}\right)\right\}\right) .
$$

Let $\Pi_{0}$ denote the identity operator and define inductively

$$
\Pi_{m+1}=E_{\Lambda_{m}} \circ \Pi_{m}, \quad \text { where } \Lambda_{m}=\Lambda_{l} \text { if } m \equiv l \bmod 4 \text {, }
$$

where $E_{\Lambda}$ is an operator defined by

$$
E_{\Lambda} f(\omega) \equiv \mu_{\Lambda}^{\omega}(f) .
$$

Then we set for any function $f$ and positive integer $m$,

$$
f_{m}^{2} \equiv \Pi_{m} f_{m-1}^{2} \text {. }
$$


Using this notation, for any $N \in \mathbb{N}$ we have

$$
\begin{aligned}
& E_{\Lambda}\left[f^{2} \log \left(\frac{f^{2}}{E_{\Lambda}\left[f^{2}\right]}\right)\right]=\sum_{m=0}^{N-1} E_{\Lambda}\left[E_{\Lambda_{m}} f_{m}^{2} \log \left(\frac{f_{m}^{2}}{E_{\Lambda_{m}}\left[f_{m}^{2}\right]}\right)\right]+E_{\Lambda}\left[f_{N}^{2} \log \left(\frac{f_{N}^{2}}{E_{\Lambda}\left[f^{2}\right]}\right)\right] \\
& \quad \leqq 2 \mathbf{c}_{L} \sum_{m=1}^{N} E_{\Lambda}\left[\left|\nabla_{\Lambda_{m}} f_{m}\right|^{2}\right]+E_{\Lambda}\left[f_{N}^{2} \log \left(\frac{f_{N}^{2}}{E_{\Lambda}\left[f^{2}\right]}\right)\right] .
\end{aligned}
$$

We need to estimate every term in the sum on the right-hand side of (83). The strategy of the proof relies on some sweeping out relations as proved in Lemma 3.14 of [35], that we now recall:

For $Y \in \mathscr{F}$, define $\rho_{Y}: \Omega^{2} \rightarrow(0, \infty)$ by:

$$
\rho_{Y}(\tilde{\omega} \mid \omega)=\frac{\exp \left\{-U_{\mathbf{J}, Y}\left(\tilde{\omega} \bullet \bullet_{Y} \omega\right)\right\}}{Z_{\mathbf{J}, Y}^{\omega}} .
$$

Next, for $j \in Y^{c}$ and $x \in \mathbf{M}$, we set

$$
R_{Y, j}(\omega \mid x)=\frac{\rho_{Y}\left(\cdot \mid x \circ_{j} \omega_{\{j\}^{c}}\right)}{\rho_{Y}(\cdot \mid \omega)}
$$

where $x \circ_{j} \omega_{\{j\}^{c}}$ is a configuration in $\Omega$ which coincides with $\omega$ on $\Lambda \backslash\{j\}$ and has $j^{\text {th }}$ coordinate equal to $x$. Finally, for $\emptyset \neq \tilde{Y} \subset Y$, define:

$$
\begin{gathered}
\mathscr{R}(Y, \tilde{Y}, j)=\sup \left\{\left|E_{\tilde{Y}}\left[R_{Y, j}(\cdot \mid x)\right]\left(\omega^{\prime}\right)-E_{\tilde{Y}}\left[R_{Y, j}(\cdot \mid x)\right]\left(\omega^{\prime \prime}\right)\right|:\right. \\
\left.x \in \mathbf{M} \text { and }\left.\left(\omega^{\prime}, \omega^{\prime \prime}\right) \in \Omega^{2} \omega^{\prime}\right|_{Y^{c}}=\left.\omega^{\prime \prime}\right|_{Y^{c}}\right\} .
\end{gathered}
$$

With this notation we have the following lemma.

Lemma 5.1. For any set $Y \in \mathscr{F}$ and any positive function $f \in \mathscr{C}^{1}$ with the property that, for each $\omega \in \Omega$, the function $f_{Y}(\cdot \mid \omega)$ is localized in $Y \backslash \tilde{Y}$, one has that:

$$
\begin{aligned}
\left|\nabla_{j}\left(E_{Y}\left[f^{2}\right]\right)^{\frac{1}{2}}\right| \leqq & 2 e^{2 h_{\Lambda}}\left(E_{Y}\left[\left|\nabla_{j} f\right|^{2}\right]^{\frac{1}{2}}+E_{j} \circ E_{Y}\left[\left|\nabla_{j} f\right|^{2}\right]^{\frac{1}{2}}\right) \\
& +2 \mathscr{R}(Y, \tilde{Y}, j)\left(E_{Y}[f, f]\right)^{\frac{1}{2}}
\end{aligned}
$$

where we have set

$$
h_{\Lambda} \equiv \sup _{j \in \Lambda}\left\|\tilde{\Phi}^{(j)}(\mathbf{J}, \cdot)\right\|_{1}
$$

As a consequence of Lemma 5.1, we can bound the terms $E_{\Lambda}\left[\left|\nabla_{\Lambda_{m}} f_{m}\right|^{2}\right]$ from the sum on the right-hand side of (83). Indeed, if for $X \in \mathscr{F}$ we set

$$
\mathbf{m}_{X}=\inf _{f \perp \mathbb{1}} \frac{\mu_{X}^{\omega}\left[\left|\nabla_{X} f\right|^{2}\right]}{\mu_{X}^{\omega}[f, f]}
$$

then, we have: 
Lemma 5.2. For any function $f$, any nonnegative integer $m$, any integer $r$, and any $j \in \partial_{R} \Lambda_{m+1}$,

$$
E_{\Lambda}\left[\left|\nabla_{j} f_{m+1}\right|^{2}\right] \leqq C_{j, j} E_{\Lambda}\left[\left|\nabla_{j} f_{m}\right|^{2}\right]+\sum_{Y_{k} \subset \Lambda_{m}} \mathbb{1}_{j \in \partial Y_{k}} \sum_{i \in Y_{k}} C_{j, i}^{Y_{k}} E_{\Lambda}\left[\left|\nabla_{i} f_{m}\right|^{2}\right]
$$

where

$$
\begin{aligned}
C_{j, j} & =2^{14} e^{12 h_{\Lambda}} \\
C_{j, i}^{Y_{k}} & =2^{12} e^{12 h_{\Lambda}} \mathbf{m}_{\mathbf{B}_{j, r} \cap Y_{k}}^{-1}\left\{2^{4} e^{4 h_{A}}+\left|\partial\left(\mathbf{B}_{j, r} \cap Y_{k}\right)\right| \mathbf{m}_{Y_{k}}^{-1} \mathscr{R}\left(Y_{k}, \mathbf{B}_{j, r} \cap Y_{k}, j\right)^{2}\right\} \text { if } i \in \mathbf{B}_{j, r} \\
& =2^{12} e^{8 h_{\Lambda}} \mathbf{m}_{Y_{k}}^{-1} \mathscr{R}\left(Y_{k}, \mathbf{B}_{j, r} \cap Y_{k}, j\right)^{2} \quad \text { otherwise }
\end{aligned}
$$

with $\mathbf{B}_{j, r}$ being the ball of radius $r$ centered at the point $j$.

Proof. First we observe that by construction there is a unique $l \in \Gamma_{m}$ such that $j \in \partial_{R} Y_{l}$. Let $\Lambda_{m, l}=\bigcup_{k \in \Gamma_{m} \cap\{l\}^{c}} Y_{k}$. Since $E_{\Lambda_{m}}$ is a product measure, we have

$$
f_{m+1}^{2} \equiv \Pi_{m+1} f^{2}=E_{\Lambda_{m, l}} \circ E_{Y_{l}}\left[f_{m}^{2}\right] .
$$

Using Lemma 5.1 and the basic observation that $j$ does not belong to the boundary of $\Lambda_{m, l}$ yields

$$
\begin{aligned}
E_{\Lambda}\left[\left|\nabla_{j} f_{m+1}\right|^{2}\right] & \equiv E_{\Lambda}\left[\left|\nabla_{j}\left(\Pi_{m+1} f_{m}^{2}\right)^{\frac{1}{2}}\right|^{2}\right]=E_{\Lambda}\left[\left|\nabla_{j}\left(E_{\Lambda_{m, l}} \circ E_{Y_{l}}\left[f_{m}^{2}\right]\right)^{\frac{1}{2}}\right|^{2}\right] \\
& \leqq 2^{4} e^{4 h_{\Lambda}} E_{\Lambda}\left[\left|\nabla_{j}\left(E_{Y_{l}}\left[f_{m}^{2}\right]\right)^{\frac{1}{2}}\right|^{2}\right]
\end{aligned}
$$

(In the continuous case the coefficient before the expectation on the right-hand side would be equal to one.) Next, let $r$ be an integer number and set $\tilde{Y}=\left\{i \in Y_{l}\right.$ : $|i-j|<r\}$ and $F=\left(E_{\tilde{Y}}\left[f_{m}^{2}\right]\right)^{\frac{1}{2}}$. Then, again by (85), but this time applied to $F$ and $Y=Y_{l}$, we get:

$$
\begin{aligned}
E_{\Lambda}\left[\left|\nabla_{j}\left(E_{Y_{l}}\left[f_{m}^{2}\right]\right)^{\frac{1}{2}}\right|^{2}\right] & =E_{\Lambda}\left[\left|\nabla_{j}\left(E_{Y_{l}}\left[F^{2}\right]\right)^{\frac{1}{2}}\right|^{2}\right] \\
& \leqq 2^{5} e^{4 h_{\Lambda}} E_{\Lambda}\left[\left|\nabla_{j} F\right|^{2}\right]+2^{3} \mathscr{R}\left(Y_{l}, \tilde{Y}, j\right)^{2} E_{\Lambda}\left[E_{Y_{l}}[F, F]\right] \\
& \leqq 2^{5} e^{4 h_{\Lambda}} E_{\Lambda}\left[\left|\nabla_{j} F\right|^{2}\right]+2^{3} \mathscr{R}\left(Y_{l}, \tilde{Y}, j\right)^{2} \mathbf{m}_{Y_{l}}^{-1} E_{\Lambda}\left[\left|\nabla_{Y_{l}} F\right|^{2}\right] .
\end{aligned}
$$

At the same time,

$$
E_{\Lambda}\left[\left|\nabla_{j} F\right|^{2}\right] \leqq 2^{5} e^{4 h_{\Lambda}} E_{\Lambda}\left[\left|\nabla_{j} f_{m}\right|^{2}\right]+2^{3} \mathscr{R}(\tilde{Y}, \emptyset, j)^{2} \mathbf{m}_{\tilde{Y}}^{-1} E_{\Lambda}\left[\left|\nabla_{\tilde{Y}} f_{m}\right|^{2}\right]
$$

and

$$
E_{\Lambda}\left[\left|\nabla_{Y_{l}} F\right|^{2}\right] \leqq \sum_{i \in Y_{l} \backslash \tilde{Y}}\left(2^{5} e^{4 h_{\Lambda}} E_{\Lambda}\left[\left|\nabla_{i} f_{m}\right|^{2}\right]+2^{3} \mathscr{R}(\tilde{Y}, \emptyset, i)^{2} \mathbf{m}_{\tilde{Y}}^{-1} E_{\Lambda}\left[\left|\nabla_{\tilde{Y}} f_{m}\right|^{2}\right]\right)
$$

Finally, since it is clear that

$$
\mathscr{R}(\tilde{Y}, \emptyset, i) \leqq \mathbb{1}_{i \in \partial_{R} \tilde{Y}} 4 e^{4 h_{\Lambda}},
$$

it is not hard to deduce Lemma 5.2 from (90), (91), (92), (93). 
Thus, the crucial point now is to bound the coefficients $C_{i, j}$. For this we need to bound the spectral gap coefficients $\mathbf{m}_{Y_{l}}$ from below and the quantities $\mathscr{R}$ from above for an appropriate choice of size $L$ of our cubes. This is the subject of the following subsection.

5.1. Some Almost Sure Bounds. For a positive integer $n$, let $\Lambda=\Lambda^{n}$ to be the cube $[-n,+n]^{2}$. We choose $r=r_{n} \equiv r_{0} \log n$ and $L=L_{n} \equiv L_{0} \log n$, with $L_{0} \equiv 2^{3} r_{0}$, for some small $r_{0}>0$ to be specified later. When necessary we will use $\Gamma_{l}^{n}, \Lambda_{m}^{n}, Y_{k}^{n}$, $\tilde{Y}_{k}^{n}$ to denote the sets constructed above with $\Lambda=\Lambda^{n}, r=r_{n}$ and $L=L_{n}$.

We first prove the almost sure bound on the random coefficients

$$
\mathscr{R}_{n}^{r_{0}}(l, j) \equiv \mathscr{R}\left(Y_{l}^{n}, B_{j, r_{n}}, j\right) .
$$

Bound on $\mathscr{R}_{n}(l, j)$.

Lemma 5.3. Suppose the conditions $(\mathbb{H} 1)$ and $(\mathbb{H} 2)$ are satisfied. There exists a positive function $\bar{r}_{0}\left(p_{1}\right), \bar{r}_{0}\left(p_{1}\right) \rightarrow 0$ when $p_{1} \rightarrow 0$, a function $\alpha \equiv \alpha\left(J_{0}\right) \in(0,1)$, $\alpha\left(J_{0}\right) \rightarrow 0$ when $J_{0} \rightarrow 0$, and a constant $D \in(0, \infty)$, such that for any $r_{0} \geqq \bar{r}_{0}\left(p_{1}\right)$, for any $J_{0}$ small enough, for any $\varepsilon \in(0,1)$, for almost every $\mathbf{J} \in \mathbb{J}$ there exists $N(\mathbf{J}) \in \mathbb{N}$, so that for any $n \geqq N(\mathbf{J})$, any $l \in \cup_{k} \Gamma_{k}^{n}$, we have:

$$
\mathscr{R}_{n}^{r_{0}}(l, j) \leqq D e^{4 h_{\Lambda^{n}}} \alpha^{r_{0} \log n} \leqq D n^{\varepsilon} \alpha^{r_{0} \log n}
$$

Proof. Let $\tilde{Y} \subset Y$ be finite subsets of $\mathbb{Z}^{2}$ and recall that from the definition of $R_{Y, j}$ we have

$$
R_{Y, j}(\omega \mid x)=\frac{Z_{\mathbf{J}, Y}^{\left(x \circ \omega_{\{,\}^{c}}\right)}}{Z_{\mathbf{J}, Y}^{\omega}} \exp \left\{-\left(\sum_{\substack{X \in \mathscr{F} \\ X \ni j}} \Phi_{X}\left(\mathbf{J}, x \circ_{j} \omega_{\{j\}^{c}}\right)-\Phi_{X}(\mathbf{J}, \omega)\right)\right\} .
$$

Hence for any $j \in \partial_{R} Y$,

$$
\begin{aligned}
\mathscr{R}(Y, \tilde{Y}, j) \leqq & 2 e^{2 h_{Y}} \sup \left\{\mid E_{\tilde{Y}}\left[\exp \left\{\sum_{X: j \in X}\left(\Phi_{X}(\omega)-\Phi_{X}\left(x \circ_{j} \omega_{\{j\}^{c}}\right)\right)\right\}\right]\right. \\
& -E_{\tilde{Y}}\left[\exp \left\{\sum_{X: j \in X}\left(\Phi_{X}(\bar{\omega})-\Phi_{X}\left(x \circ_{j} \bar{\omega}_{\{j\}^{c}}\right)\right)\right\}\right] \mid \\
& \left.: x \in \mathbf{M} \text { and }\left.(\omega, \bar{\omega}) \in \Omega^{2} \omega\right|_{Y^{c}}=\left.\bar{\omega}\right|_{Y^{c}}\right\} .
\end{aligned}
$$

Furthermore, $\sum_{X: j \in X}\left(\Phi_{X}(\omega)-\Phi_{X}\left(x \circ_{j} \omega_{\{j\}^{c}}\right)\right)$ is localised in $\mathbf{B}_{j, R} \subset \mathbf{B}_{j, r_{n}}$ for large $n$. Thus, using clustering properties in the small couplings regions proven in [6] (see Theorem 2.1), one finds that

$$
\mathscr{R}(Y, \tilde{Y}, j) \leqq 4 e^{4 h_{Y}} \sum_{W \in \mathscr{W}(j, \tilde{Y})} \prod_{X \in W} \gamma_{X}
$$

where $\mathscr{W}(j, \tilde{Y})$ denotes the set of the connected paths $W=\left(X_{1}, \ldots, X_{p}\right)$ of (mutually different) sets intersecting $\tilde{Y}$ with diameter less than $R$ and such that $j \in X_{1}$, 
$X_{i} \cap X_{i+1} \neq \emptyset, \tilde{Y}^{c} \cap X_{p} \neq \emptyset$ and

$$
\gamma_{X}= \begin{cases}e^{4 J_{0}}-1 & \text { if }\left|\Phi_{X}\right| \leqq J_{0} \\ 1 & \text { otherwise }\end{cases}
$$

Using our assumption $\mathbb{H} 1$ about the interaction we have the following simple almost sure bound on the first factor from the right-hand side (96):

$$
h_{\Lambda^{n}} \leqq \frac{\varepsilon}{4} \log n
$$

with any $\varepsilon \in(0,1)$ for all sufficiently large $n$ (dependent on $\mathbf{J}$ ). Thus we need only to get an almost sure bound of the sum from the right-hand side of (96) in the case where $Y=Y_{l}^{n}$ and $\tilde{Y}=\mathbf{B}_{j, r_{n}} \cap Y_{k}$. As observed in [6] this boils down to showing that with probability one in every term $\prod_{X \in W} \gamma_{X}$ a large number of factors is smaller than one. This is the content of the following lemma (stated in any dimension).

Lemma 5.4. Let

$$
\lambda_{j}(r)=\inf _{W \in \mathscr{W}\left(j, \mathbf{B}_{,}, r\right.} \frac{\#\left\{X \in W:\left\|\Phi_{X}(\mathbf{J}, \cdot)\right\|_{u} \leqq J_{0}\right\}}{\#\{X \in W\}} .
$$

Then, for any $\eta \in(0,1)$, there exists a positive function $\bar{r}_{0}^{\eta}\left(p_{1}, \eta, d\right), \bar{r}_{0}^{\eta}\left(p_{1}, \eta, d\right) \rightarrow 0$ when $p_{1} \rightarrow 0$, such that, for any $r_{0} \geqq \bar{r}_{0}^{\eta}\left(p_{1}, \eta, d\right)$ :

$$
\mathbb{E}\left\{\bigcap_{k \in \mathbb{N}} \bigcup_{n \geqq k}\left\{\inf _{j \in \Lambda^{n}} \lambda_{j}\left(r_{0} \log n\right) \geqq \eta\right\}\right\}=1,
$$

provided that $p_{1} \equiv \sup _{X} \mathbb{E}\left\{\left\|\Phi_{X}\right\|_{u} \geqq J_{0}\right\}<p_{c}^{b}(d, R)$ with some $p_{c}^{b}(d, R) \in(0,1)$ sufficiently small.

In the special case $R=1$ this is a result of Kesten [25]. A proof of Lemma 5.4 will be given below (only for the readers' convenience, as the idea is similar to the one in [25]). Now we can use it to complete the proof of Lemma 5.3 as follows. By Lemma 5.4 we have with probability one for $n$ sufficiently large an estimate

$$
\sum_{W \in \mathscr{W}\left(j, \mathbf{B}_{J}, r_{n}\right)} \prod_{X \in W} \gamma_{X} \cdot \leqq \sum_{l \geqq\left[\frac{r_{n}}{R}\right]} C(R)^{l}\left(e^{4 J_{0}}-1\right)^{\eta l} \leqq \frac{1}{\alpha^{R}\left(1-\alpha^{R}\right)} \alpha^{r_{n}}
$$

where

$$
\alpha \equiv\left(C(R)\left(e^{4 J_{0}}-1\right)^{\eta}\right)^{\frac{1}{R}}
$$

with some constant $C(R) \in(0, \infty)$ dependent only on the range of the interaction (for a given dimension of the lattice). This together with (97) implies the desired estimate of Lemma 5.3.

Now we prove Lemma 5.4. 
Proof of Lemma 5.4. We will show that the opposite event has probability zero, i.e. that for some $\eta \in(0,1)$, we have

$$
\mathbb{E}\left\{\bigcap_{k \in \mathbb{N}} \bigcup_{n \geqq k}\left\{\exists j \in \Lambda^{n}: \lambda_{j}\left(r_{n}\right)<\eta\right\}\right\}=0 .
$$

For this, using the definition of $\lambda_{j}(r)$ and that the interaction is of finite range $R$, we observe that for a given $\eta \in(0,1)$, we have:

$$
\begin{aligned}
\mathbb{E}\{ & \left.\lambda_{j}(r)<\eta\right\} \\
\equiv & \mathbb{E}\left\{\exists X_{1}, \ldots, X_{k}: j \in X_{1}, X_{i} \cap X_{i+1} \neq \emptyset, X_{k} \cap \mathbf{B}_{j, r}^{c} \neq \emptyset, k \geqq\left[\frac{r}{R}\right], X_{i} \neq X_{j},\right. \\
& \left.\sum_{i=1}^{k} \mathbb{1}_{\left\|\Phi_{X}\right\|_{u}>J_{0}} \geqq(1-\eta) k\right\} .
\end{aligned}
$$

Since with some constants $C(R, d) \in(0, \infty)$

$$
\#\left(\exists X_{1}, \ldots, X_{k}: j \in X_{1}, X_{i} \cap X_{i+1} \neq \emptyset, X_{k} \cap \mathbf{B}_{j, r}^{c} \neq \emptyset\right) \leqq C(R, d)^{k},
$$

using the Tchebyshev inequality, for any positive real number $A$, we have:

$$
\mathbb{E}\left\{\lambda_{j}(r)<\eta\right\} \leqq \sum_{k \geqq\left[\frac{r}{R}\right]} C(R, d)^{k} e^{-A(1-\eta) k}\left(\sup _{X} \mathbb{E}\left[e^{A \mathbb{1}_{\left\|\Phi_{X}\right\|_{u}>J_{0}}}\right]\right)^{k} .
$$

But, if $p_{1}=\mathbb{E}\left\{\left\|\Phi_{X}\right\|_{u}>J_{0}\right\}$,

$$
\sup _{X} \mathbb{E}\left[e^{A \mathbb{1}_{\left\|\Phi_{X}\right\|_{u}>J_{0}}}\right]=e^{A} p_{1}+1-p_{1} .
$$

As a consequence, if $p_{1}$ is small enough, we can pick $\eta$ and $A$ so that:

$$
\Delta(A) \equiv \Delta\left(R, p_{1}, A, \eta\right) \equiv C(R)\left(e^{A} p_{1}+1-p_{1}\right) e^{-A(1-\eta)}<1 .
$$

For example, if $p_{1}<\frac{1}{2 C(R)}$, and if we choose $A=-\log p_{1}$, we get that, for any $\eta$ :

$$
\Delta \equiv \Delta\left(-\log p_{1}\right)=C(R, d) p_{1}^{1-\eta}\left(2-p_{1}\right) \leqq 2 C(R, d) p_{1}^{1-\eta},
$$

which is smaller than 1 if $\eta$ is small enough. Using (99), we get

$$
\sum_{n \in \mathbb{N}} \mathbb{E}\left\{\exists j \in \Lambda^{n}: \lambda_{j}\left(r_{0} \log n\right)<\eta\right\} \leqq \frac{1}{\Delta(1-\Delta)} \sum_{n \in \mathbb{N}}\left|\Lambda^{n}\right| \Delta^{\frac{r_{n}}{R}} .
$$

Since by our choice $\left|\Lambda^{n}\right|=(2 n+1)^{d}$ and $r_{n}=r_{0} \log n$ we get

$$
\sum_{n \in \mathbb{N}} \mathbb{E}\left\{\exists j \in \Lambda^{n}: \lambda_{j}<\eta\right\}<\infty,
$$

provided that

$$
\frac{r_{0}}{R} \log \Delta+d<-1 \text {. }
$$

This clearly can be satisfied when $p_{1}$ is small enough. Now, if (103) is true, we can use the Borel-Cantelli lemma to conclude that (98) is true. This concludes the proof of Lemma 5.4 . 
Remark. If $p_{1}<(1 / 2 C(R, d))$, it follows from (100) that, for any $\eta$ in $(0,1)$, such that $2 C(R, d) p_{1}^{1-\eta}<1$, we can choose $r_{0}$ as any real number in $\left(\bar{r}_{0}\left(p_{1}\right),+\infty\right)$ with

$$
\bar{r}_{0} \equiv \bar{r}_{0}\left(p_{1}, \eta, d\right)=\frac{R(d+1)}{-\log 2 C(R, d) p_{1}^{1-\eta}} .
$$

It is clear that $\bar{r}_{0}\left(p_{1}\right)$ goes to zero when $p_{1} \rightarrow 0$.

Bound on the spectral gap coefficients. We will show the following almost sure lower bound for spectral gap:

Lemma 5.5. There exists a positive constant $\tau$ such that:

$$
\mathbb{E}\left\{\bigcup_{N} \bigcap_{n \geqq N}\left\{\forall k \in \bigcup_{l} \Gamma_{l}^{n}, \mathbf{m}_{Y_{k}^{n}} \geqq \exp (-\tau \log n)\right\}\right\}=1
$$

and

$$
\mathbb{E}\left\{\bigcup_{N} \bigcap_{n \geqq N}\left\{\forall j \in \Lambda^{n}, \mathbf{m}_{\mathbf{B}_{\jmath, n}} \geqq \exp (-\tau \log n)\right\}\right\}=1 .
$$

Remark. One can choose

$$
\tau \in\left(\inf _{s>0}\left(2^{2} R L_{0} \frac{1}{s} \log E\left[\exp \left(s\left\|\tilde{\Phi}^{(j)}\right\|_{1}\right)\right]+\frac{3}{s}\right),+\infty\right) .
$$

We remark that we can choose $\tau \equiv \tau\left(L_{0}\right) \rightarrow 0$ as $L_{0} \rightarrow 0$.

Proof. We first recall the standard spectral gap lower bound: For any $\omega \in \Omega$ and any cube $Y$ we have

$$
\inf _{f \in L_{2}\left(\mu_{\mathbf{J}, Y}^{\omega}\right)} \frac{\mu_{\mathbf{J}, Y}^{\omega}\left|\nabla_{Y} f\right|^{2}}{\mu_{\mathbf{J}, Y}^{\omega}(f, f)} \geqq\left(|Y| \sup _{i \in Y} \exp \left(8 \sum_{X \cap V^{\prime} \neq \emptyset}\left\|\Phi_{X}\right\|_{u}\right)\right)^{-1}
$$

where $V^{i, n}=\left\{j \in Y_{k}:\left|j_{1}-i_{1}\right| \leqq R\right\}$. We have also the following large deviation estimate.

Lemma 5.6. There exists a finite constant $\tau$, such that:

$$
\mathbb{E}\left\{\bigcup_{N} \bigcap_{n \geqq N}\left\{\forall k \in \bigcup_{l} \Gamma_{l}^{n}, L_{n}^{2} \sup _{i \in Y_{k}^{n}} \exp \left(8 \sum_{X \cap V^{\prime, n} \neq \emptyset}\left\|\Phi_{X}\right\|_{u}\right) \leqq \exp (\tau \log n)\right\}\right\}=1 .
$$

Proof. Indeed, by Borel Cantelli's Lemma, it is enough to show that:

$$
\sum_{n} \mathbb{E}\left\{\exists k \in \bigcup_{l} \Gamma_{l}^{n}, L_{n}^{2} \sup _{i \in Y_{h}^{n}} \exp \left(8 \sum_{X \cap V^{l, n} \neq \emptyset}\left\|\Phi_{X}\right\|_{u}\right)>\exp (\tau \log n)\right\}<\infty
$$


for some constant $\tau \in(0, \infty)$. But, Tchebyshev's inequality implies that for any $s>0$ :

$$
\begin{aligned}
& \mathbb{E}\left\{\exists k \in \bigcup_{l} \Gamma_{l}^{n}, \sup _{i \in Y_{k}^{n}} \exp \left(8 \sum_{X \cap V^{i, n} \neq \emptyset}\left\|\Phi_{X}\right\|_{u}\right)>\frac{\exp (\tau \log n)}{\left(L_{0} \log n\right)^{2}}\right\} \\
& \leqq \text { const. }(\log n)^{2 s} n^{2} \exp \left\{\left(-s \tau+4 R L_{0} \log E\left[\exp \left(8 s\left\|\tilde{\Phi}^{(j)}\right\|_{1}\right)\right]\right) \log n\right\},
\end{aligned}
$$

where we used in the last line the fact that $\left|V^{i, n}\right| \leqq 2 R\left(2 L_{0} \log n+2 R\right)$ and the invariance by translation of the law of $\left\|\tilde{\Phi}^{(j)}\right\|_{1}$; const denotes a positive constant independent of $n \in \mathbb{N}$.

As a consequence, if we choose $\tau>2^{2} R L_{0} \frac{1}{s} \log E\left[\exp \left\{s\left\|\tilde{\Phi}^{(j)}\right\|_{1}\right\}\right]+\frac{3}{s}$, for some $s$, we get Lemma 5.6. Using Lemma 5.6 and the standard spectral gap lower bound we obtain the first point of Lemma 5.5 with the constant $\tau$ given in Lemma 5.6.

It is clear that we can apply the same strategy to prove the second point of Lemma 5.5.

5.2. Almost Sure Local Logarithmic Sobolev Inequality. As a consequence of Lemmas 5.2, 5.3 and 5.5 we get the following estimate:

Lemma 5.7. Suppose $(\mathrm{H} 2)$ is true with some sufficiently small $J_{0} \in(0, \infty)$. Then for almost every coupling $\mathbf{J}$, there exists an integer $N(\mathbf{J})$, so that for any $n \geqq N(\mathbf{J})$, any integer $m$ and any $j \in \partial \Lambda_{m+1}^{n}$, and all functions $f \in \mathscr{C}^{1}$,

$$
\begin{aligned}
E_{\Lambda^{n}}\left[\left|\nabla_{j}\left(\Pi_{m+1} f^{2}\right)^{\frac{1}{2}}\right|^{2}\right] \leqq & K_{n}\left\{\sum_{i:|i-j| \leqq r_{0} \log n} E_{\Lambda^{n}}\left[\left|\nabla_{i}\left(\Pi_{m} f^{2}\right)^{\frac{1}{2}}\right|^{2}\right]\right. \\
& \left.+\alpha^{r_{0} \log n} \sum_{i \cdot|l-j| \leqq 2 L_{0} \log n+3 R} E_{\Lambda^{n}}\left[\left|\nabla_{i}\left(\Pi_{m} f^{2}\right)^{\frac{1}{2}}\right|^{2}\right]\right\},
\end{aligned}
$$

where

$$
K_{n}=2^{14} D e^{16 h_{\Lambda^{n}}} e^{\tau \log n}\left(1+2 R r_{0} \log n \alpha^{r_{0} \log n} e^{\tau \log n}\right) .
$$

We now follow the end of the proof of (3.13) given in [35]. With $L_{0}=2^{3} r_{0}$, by induction we find that:

Lemma 5.8. For almost every coupling $\mathbf{J}$, there exists an integer number $N(\mathbf{J})$ such that, for any $n \geqq N(\mathbf{J})$, any $j \in \partial \Lambda_{m+1}^{n}$ and for all functions $f \in \mathscr{C}^{1}$, we have

$$
E_{\Lambda^{n}}\left[\left|\nabla_{\Lambda^{n}}\left(\Pi_{4} f^{2}\right)^{\frac{1}{2}}\right|^{2}\right] \leqq \Xi_{n} E_{\Lambda^{n}}\left[\left|\nabla_{\Lambda^{n}} f\right|^{2}\right]
$$

where

$$
\Xi_{n} \equiv\left(4 K_{n}\left(2 L_{0} \log n+3 R\right)\right)^{12} \alpha^{r_{0} \log n} .
$$

Now suppose that $r_{0} \log \alpha+12 \tau<0$, i.e. if $J_{0}$ is small enough so that there exists an $\eta>0$ and a $s>0$ such that:

$$
r_{0} \eta \log \left(e^{4 J_{0}}-1\right)+12\left(2^{2} R L_{0} \frac{1}{s} \log E\left[\exp \left\{s\left\|\tilde{\Phi}^{(j)}\right\|_{1}\right\}\right]+\frac{3}{s}\right)<0,
$$


and so $\Xi_{n} \in(0,1)$. Then we find that, for $n$ large enough:

$$
\sum_{m \geqq 4} E_{\Lambda^{n}}\left[\left|\nabla_{\Lambda_{m+1}} f_{m}\right|^{2}\right] \leqq \frac{\Xi_{n}}{1-\Xi_{n}} \sum_{m<4} E_{\Lambda^{n}}\left[\left|\nabla_{\Lambda^{n}} f_{m}\right|^{2}\right] .
$$

But we can also use Lemma 5.7 to see that, when $m \leqq 3$ :

$$
E_{\Lambda^{n}}\left[\left|\nabla_{\Lambda^{n}} f_{m}\right|^{2}\right] \leqq K_{n} E_{\Lambda^{n}}\left[\left|\nabla_{\Lambda^{n}} f_{m-1}\right|^{2}\right]
$$

so that, again by induction, we conclude that for $n$ large enough:

$$
\sum_{m \geqq 0} E_{\Lambda^{n}}\left[\left|\nabla_{\Lambda_{m+1}}\left(f_{m}^{2}\right)^{\frac{1}{2}}\right|^{2}\right] \leqq 3 \frac{1+K_{n}^{3}}{1-\Xi_{n}} E_{\Lambda^{n}}\left[\left|\nabla_{\Lambda^{n}} f\right|^{2}\right] .
$$

Hence (83) applied with $\Lambda=\Lambda^{n}$ and $L=L_{n} \equiv\left[L_{0} \log n\right]$, gives that

$$
E_{\Lambda^{n}}\left[f^{2} \log \left(\frac{f^{2}}{E_{\Lambda^{n}}\left[f^{2}\right]}\right)\right] \leqq \overline{\mathbf{c}}_{\Lambda^{n}} E_{\Lambda^{n}}\left[\left|\nabla_{\Lambda^{n}} f\right|^{2}\right],
$$

where

$$
\overline{\mathbf{c}}_{\Lambda^{n}} \equiv \mathbf{c}_{L_{n}} 3 \frac{1+K_{n}^{3}}{1-\Xi_{n}} .
$$

Finally, we can bound the logarithmic Sobolev coefficient $\mathbf{c}_{L_{n}}$ in terms of the spectral gap coefficients on the cubes $Y_{k}^{n}$ and of the logarithmic Sobolev coefficient $\mathbf{c}_{0}$ on $\left(\mathbf{M}, v_{\mathbf{M}}\right)$ as follows.

\section{Lemma 5.9.}

$$
\begin{aligned}
\mathbf{c}_{L_{n}} & \leqq \sup _{k \in \cup_{l} \Gamma_{l}^{n}}\left\{2 \mathbf{c}_{0}+\frac{1}{\mathbf{m}_{Y_{k}^{n}}} 2 \sup _{X \in Y_{k}}\left\|\tilde{\Phi}^{(j)}\right\|_{1}^{2}\left|Y_{k}\right| \mathbf{c}_{0}+\sup _{j \in Y_{k}}\left\|\tilde{\Phi}^{(j)}\right\|_{1}\left|Y_{k}\right|+1\right\} \\
& \leqq C(R)\left(1+\mathbf{c}_{0}\right)\left(1+h_{\Lambda^{n}}\right)\left(1+(\log n)^{3}\right) e^{\tau \log n}
\end{aligned}
$$

for a finite constant $C(R)$.

Now let us recall that for large $n$,

$$
h_{\Lambda^{n}} \leqq \varepsilon \log n
$$

with arbitrary small $\varepsilon \in(0,1)$. Let us note also that all the positive constants $\alpha \equiv \alpha\left(J_{0}\right), r_{0} \equiv r_{0}\left(p_{1}\right), L_{0} \equiv 2^{3} r_{0}$ and the constant $\tau \equiv \tau\left(p_{1}\right)$ can be made arbitrarily small by choosing $p_{1}$ sufficiently small. Therefore, using the formula for $K_{n}$, $\Xi_{n}$ and $\mathbf{c}_{L_{n}}$, we conclude that there is a constant $\vartheta \equiv \vartheta\left(J_{0}, p_{1}\right) \rightarrow 0$ as $J_{0}, p_{1} \rightarrow 0$ such that for all $n \geqq N(\mathbf{J})$ we have

$$
\overline{\mathbf{c}}_{\Lambda^{n}} \leqq \bar{C}(\mathbf{J}) n^{\vartheta}
$$

with some positive constant $\bar{C}(\mathbf{J})$. In particular by choosing $J_{0} \geq 0$ and $p_{1}>0$ sufficiently small, we get the bound given above satisfied with $\bar{\vartheta} \in(0,1)$. Now taking into account the assumption (IHD), which implies the almost sure bound on the growth of $a_{X+i}^{-1}$, one easily arrives at the result of Theorem 4.2 with

$$
c_{n} \equiv \mathbf{c}_{\mathbf{J}, \Lambda^{n}} \leqq C(\mathbf{J}) n^{\vartheta}
$$

with some positive constant $C(\mathbf{J})$ independent of $n \in \mathbb{N}$. This ends the proof of Theorem 4.2. 


\section{References}

1. Aizenman, M., Holley, R.: Rapid Convergence to Equilibrium of Stochastic Ising Models in the Dobrushin-Shlosman Regime. In: Percolation Theory and Ergodic Theory of Infinite Particle Sytems, ed. Kesten, H., Berlin-Heidelberg-New York: Springer-Verlag 1987, pp. 1-11

2. Ben Arous, G., Guionnet, A.: Large Deviations for Langevin Spin Glass Dynamics. Probab. Theory Relat. Fields 102, 455-509 (1995)

3. Ben Arous, G., Guionnet, A.: Annealed and Quenched Propagation of Chaos for Asymmetric Spin Glass Dynamics. Submitted to Probab. Theory Relat. Fields

4. Ben Arous, G., Guionnet, A.: Symmetric Langevin spin glass dynamics. Submitted to Annals of Probab.

5. Beretti, A.: Some Properties of Random Ising models. J. Stat. Phys. 38, 438-496 (1985)

6. Von Dreyfus, H., Klein, A., Perez, J.F.: Taming Griffiths singularities: Infinite differentiability of quenched correlation functions. Commun. Math. Phys. 170, 21-39 (1995)

7. Dobrushin, R.L., Bassalygo, L.A.: Uniqueness of a Gibbs Field with random potential - An elementary approach. Theory Probab. Appl. 31, No 4, 572-589 (1986)

8. Dobrushin, R.L., Martirosyan, M.R.: Possibility of the High Temperature Phase Transitions Due to the Many-Particle Nature of the Potential. Theor. Math. Phys. 75, 443-448 (1988)

9. Dobrushin, R.L., Shlosman, S.: Constructive criterion for the uniqueness of Gibbs field. In: Statistical Physics and Dynamical Systems, Rigorous Results, Eds. Fritz, J., Jaffe, A., and Szasz, D., Basel-Boston: Birkhäuser, 1985, pp. 347-370

10. Dobrushin, R.L., Shlosman, S.: Completely analytical Gibbs fields. In: Statistical Physics and Dynamical Systems, Rigorous Results, Eds. Fritz, J., Jaffe, A., Szasz, D., Basel-Boston: Birkhäuser, 1985, pp. 371-403

11. Dobrushin, R.L., Shlosman, S.: Completely analytical interactions: Constructive description. J. Stat. Phys. 46, 983-1014 (1987)

12. Dunlop, F.: Correlation Inequalities for multicomponent Rotators. Commun. Math. Phys. 49, 247-256 (1976)

13. Fröhlich, J.: Mathematical Aspects of the Physics of Disordered Systems. In: Critical Phenomena, Random Systems, Gauge Theories, Eds. Osterwalder, K., Stora, R., Amsterdam: Elsevier, 1986

14. Fröhlich, J., Zegarlinski, B.: The High-Temperature Phase of Long-Range Spin Glasses. Commun. Math. Phys. 110, 121-155 (1987)

15. Fröhlich, J., Zegarlinski, B.: Some Comments on the Sherrington-Kirkpatrick Model of Spin Glasses. Commun. Math. Phys. 112, 553-566 (1987)

16. Fröhlich, J., Zegarlinski, B.: Spin Glasses and Other Lattice Systems with Long-Range Interactions. Commun. Math. Phys. 120, 665-688 (1989)

17. Van Enter, A.C.D., Zegarlinski, B.: A Remark on Differentiability of the Pressure Functional. Rev. Math. Phys. 17, 959-977 (1995)

18. Fröhlich, J., Imbrie, J.Z.: Improved Perturbation Expansion for Disordered Systems: Beating Griffiths Singularities. Commun. Math. Phys. 96, 145-180 (1984)

19. Fröhlich, J., Spencer, T.: The Kosterlitz-Thouless Transition in Two-Dimensional Abelian Spin Systems and the Coulomb Gas. Commun. Math. Phys. 81, 527-602 (1981)

20. Gelis, G., Maes, C.: Percolation Techniques in Disordered Spin Flip Dynamics: Relaxation to the Unique Invariant Measure. K.U. Leuven, Preprint 1995

21. Griffiths, R.: Non-Analytic Behaviour Above the Critical Point in a Random Ising Ferromagnet. Phys. Rev. Lett. 23, 17-19 (1969)

22. Gross, L.: Logarithmic Sobolev inequalities. Am. J. Math. 97, 1061-1083 (1976)

23. Guionnet, A.: Thesis, Université Paris-Sud, March 1995

24. Holley, R., Stroock, D.: Logarithmic Sobolev inequalities and stochastic Ising models. J. Stat. Phys. 46, 1159-1194 (1987)

25. Kesten, H.: Aspect of first passage percolation. Ecole d'ete de St Flour, LNM 1180, pp. 125-264 (1986)

26. Lu, Sheng Lin., Yau, Horng-Tzer: Spectral Gap and Logarithmic Sobolev Inequality for Kawasaki and Glauber Dynamics. Commun. Math. Phys. 156, 399-433 (1993)

27. Majewski, A.W., Zegarlinski, B.: Quantum Stochastic Dynamics I: Spin Systems on a Lattice. Math. Phys. Electr. J. 1, Paper 2 (1995) 
28. Martinelli, F., Olivieri, E.: Approach to Equilibrium of Glauber Dynamics in the One Phase Region: I. The Attractive case/ II. The General Case. Commun. Math. Phys. 161, 447-486 and 487-514 (1994)

29. Martinelli, F., Olivieri, E., Shonmann, R.H.: For 2-D Lattice Spin Systems Weak Mixing Implies Strong Mixing. Commun. Math. Phys. 165, 33-47 (1994)

30. Pfister, C.-E.: Translation Invariant Equilibrium States of Ferromagnetic Abelian Lattice Systems. Commun. Math. Phys. 86, 375-390 (1982)

31. Ruelle, D.: Statistical Mechanics: Rigorous Results. New York: W.A. Benjamin Inc., 1969

32. Schonmann, R.H., Schlosman, S.B.: Complete Analyticity for 2D Ising Completed. Preprint 1995

33. Stroock, D.W., Zegarlinski, B.: The Logarithmic Sobolev inequality for Continuous Spin Systems on a Lattice. J. Funct. Anal. 104, 299-326 (1992)

34. Stroock, D.W., Zegarlinski, B.: The Equivalence of the Logarithmic Sobolev Inequality and the Dobrushin-Shlosman Mixing Condition. Commun. Math. Phys. 144, 303-323 (1992)

35. Stroock, D.W., Zegarlinski, B.: The Logarithmic Sobolev inequality for Discrete Spin Systems on a Lattice. Commun. Math. Phys. 149, 175-193 (1992)

36. Stroock, D.W., Zegarlinski, B.: The ergodic properties of Glauber dynamics. J. Stat. Phys. 81, 1007-1019 (1995)

37. Thomas, L.E.: Bound on the Mass Gap for Finite Volume Stochastic Ising Models at Low Temperature. Commun. Math. Phys. 126, 1-11 (1989)

38. Zegarlinski, B.: Interactions and Pressure Functionals for Disordered Lattice Systems. Commun. Math. Phys. 139, 305-339 (1991)

39. Zegarlinski, B.: Strong Decay to Equilibrium in One Dimensional Random Spin Systems. J. Stat. Phys. 77, 717-732 (1994)

40. Zegarlinski, B.: Spin Systems with Long-Range Interactions. Rev. Math. Phys. 6, 115-134 (1994)

41. Zegarlinski, B.: Ergodicity of Markov Semigroups. In: Proc. of the Conference: Stochastic Partial Differential Equations, Edinburgh 1994, Ed. A. Etheridge, LMS Lecture Notes 216, Cambridge, Cambridge University Press 1995, pp. 312-337 